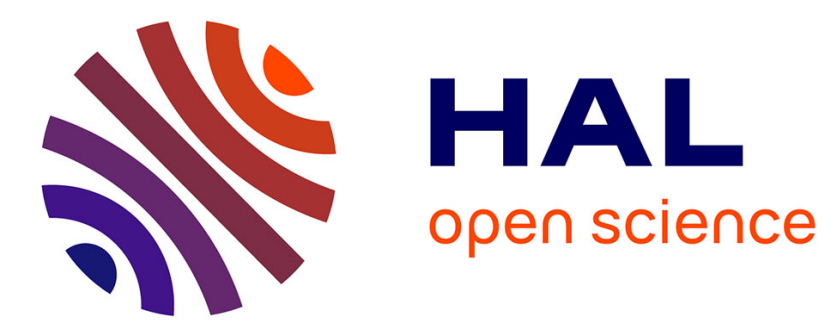

\title{
Positioning using mobile TV based on the DVB-SH standard
}

\author{
Paul Thevenon, Damien Serant, Olivier Julien, Christophe Macabiau, Michel \\ Bousquet, Lionel Ries, Stéphane Corazza
}

\section{- To cite this version:}

Paul Thevenon, Damien Serant, Olivier Julien, Christophe Macabiau, Michel Bousquet, et al.. Positioning using mobile TV based on the DVB-SH standard. Navigation, 2011, 58 (2), pp 71-90. hal-01021604

\section{HAL Id: hal-01021604 https://hal-enac.archives-ouvertes.fr/hal-01021604}

Submitted on 29 Sep 2014

HAL is a multi-disciplinary open access archive for the deposit and dissemination of scientific research documents, whether they are published or not. The documents may come from teaching and research institutions in France or abroad, or from public or private research centers.
L'archive ouverte pluridisciplinaire HAL, est destinée au dépôt et à la diffusion de documents scientifiques de niveau recherche, publiés ou non, émanant des établissements d'enseignement et de recherche français ou étrangers, des laboratoires publics ou privés. 


\title{
Positioning Using Mobile TV Based on the DVB-SH Standard
}

Thevenon Paul, TéSA-ISAE

Serant Damien, ENAC

Julien Olivier, ENAC

Macabiau Christophe, ENAC

Bousquet Michel, TéSA-ISAE

Ries Lionel, CNES

Corazza Stéphane, Thales Alenia Space

\begin{abstract}
Positioning in urban or indoor environment is a hot topic, either due to regulations such as the E911 requiring US mobile telecommunication operators to be able to locate their subscribers in case of emergency, or due to the market development, with the extension of location-based services targeting the mass market concentrated in metropolitan areas. In deep urban or indoor areas, it is generally recognized that satellite-based positioning systems are not suitable (alone) to provide a continuous, reliable and accurate position to the user. Therefore, alternative positioning techniques may be useful to complement or replace satellite positioning in these environments. This paper investigates the possibility of using a mobile TV system based on the DVB-SH standard as system of opportunity for positioning. A systemlevel modification is used in order to make emitter discrimination possible in the synchronized emitter network. Then, the signal processing steps required for pseudo-range estimation are detailed. The proposed method is simulated using realistic channel sounding measurements and provides a mean positioning error around $40 \mathrm{~m}$.
\end{abstract}

\section{Introduction}

Positioning in urban or indoor environment is currently a hot topic, either due to regulations such as the E911 requiring US mobile telecommunication operators to be able to locate their subscribers in case of emergency [1], or due to the market development, with the extension of location-based services targeting the mass market concentrated in metropolitan areas. In urban or indoor areas, it is generally recognized that satellite-based positioning systems are not suitable (alone) to provide a continuous, reliable and accurate position to the user. Therefore, alternative positioning techniques may be useful to complement or replace satellite positioning in these environments. Among others, the following systems are considered as serious candidates:

- Inertial sensors (gyro, accelerometers), magnetometers, vision-based sensors. This type of sensor is complementary to GNSS and can provide aiding in GNSS signal tracking and/or position calculation. They can also increase positioning continuity in case of short GNSS signal outage.

- Dedicated radiolocation systems. These systems are deployed in order to provide a positioning service on a given coverage. Among the wide range of existing solutions, there exist GNSS pseudolites, Ultra-Wideband (UWB) emitters, fixed RFID tags, etc. The main drawback of this type of solution is its high cost due to the deployment of extra infrastructure for a limited service coverage. 
- Systems or signals of opportunity. This type of solution consists in exploiting existing systems or signals, which have not been initially deployed for positioning. These are typically radiocommunication systems, such as mobile communication systems or broadcast systems.

This paper focuses on the last category: the systems of opportunity. A thorough analysis of the advantages and challenges associated to systems of opportunity can be found in [2]. For the sake of introducing this domain, the major advantages and challenges are summarized here.

The main advantages of systems of opportunity are their high availability in urban and indoor environments, and the possibility to provide 2 services with only one system. This means that the provision of a positioning service using a system of opportunity can be achieved at very low cost, by reusing the existing infrastructure. Also, this can also lead to the integration of positioning and telecommunication services at different levels: for example, combined services using positioning and telecommunication or a receiver sharing sub-systems between both services, which would result in a reduced cost.

The main drawback for using systems of opportunity for positioning is that their design is not optimized for positioning. Design choices for telecommunication are different than for positioning. This can be illustrated at the signal level (pseudo-random noise codes with short length, band-limited modulation, etc.) or at the system level (only one emitter covering a given zone, no synchronization between emitters, etc.). Therefore, positioning performances will be obtained through a "best effort" approach, making the most of the existing systems with as little modifications so as not to degrade the initial telecommunication service. Another major drawback comes from the fact that systems of opportunity often use terrestrial emitters. Consequently, the transmission between those emitters and a receiver in an urban environment will be affected by intense multipath, local heavy signal fading and probably non-line-of-sight propagation. This could be a problem if geometric positioning principles are used.

Several telecommunication systems have been already investigated as positioning system of opportunity. Positioning has been standardized for the $3 \mathrm{G}$ mobile systems [3]. Beside the use of assisted-GPS, positioning techniques based on the use of the serving emitter identity (Cell ID) and measurements of time difference of arrival (TDoA) of the data packets sent by several emitters have been identified. The Cell ID technique provides an accuracy which depends on the size of the emitter's coverage. It can vary between $150 \mathrm{~m}$ in cities and $30 \mathrm{~km}$ in rural areas [3]. The accuracy obtained by the TDoA depends on the emitters' configuration and on the multipath environment. In urban scenarios, the $67 \%$ position error varies between $30 \mathrm{~m}$ and $2000 \mathrm{~m}$ depending on the multipath intensity scenario [4] [5].

Broadcasting systems have also been investigated. [6] uses timing measurements of digital TV signals to obtain a position estimate with a bias of about $25 \mathrm{~m}$ and a standard deviation of $20 \mathrm{~m}$ in an indoor environment in San Francisco. One common issue of using timing measurements in these systems is that the emitters are not synchronized, thus creating a bias in the pseudo-range measurements. Therefore, reference stations have to be used in order to calculate the synchronization offset between different emitters. This offset is then used in the position calculation to correct the bias of the pseudo-range measurements. This additional infrastructure may be too costly to make this solution attractive to telecommunication system operators. [7] assesses this additional cost to be up to $170 \mathrm{M} \$$ for a German coverage.

Synchronized networks of emitters begin to appear in some standards, under the name Single Frequency Network (SFN). Depending on the synchronization level of the emitters, it may remove the need of reference stations. This feature is used for example in the Digital Audio Broadcast (DAB) digital radio standard. Trials with DAB signals have led to a mean position error of $150 \mathrm{~m}$ [8].

From this overview of the state of the art of the domain of positioning using system of opportunity, we can see that interesting solutions are emerging. Still, the limitations of the reviewed solutions, either in terms of positioning performances or required additional costs, may hamper the adoption of such solutions by service providers or system operators. In this paper, we propose to use another system of opportunity based on the mobile TV standard called "Digital Video Broadcast - Satellite to Handheld" (DVB-SH) that 
would provide good positioning performances with limited system modifications, therefore limiting the additional costs and risks taken by a service operator.

The paper is structured as follows. First, the choice of the DVB-SH system is discussed, showing both the advantages and the challenges associated with this choice. Secondly, the overall positioning strategy will be presented. In the third part, the DVB-SH signal model will be presented. In the fourth, the particular propagation channel created by the urban environment in a SFN will be presented, along with a systemlevel modification to allow emitter discrimination and identification. In the fifth part, the pseudo-range estimation method will be described. In the sixth part, the overall pseudo-range estimation method will be simulated using a realistic SFN Channel Impulse Response obtained from measurements. The article will conclude on the reachable positioning performances with the presented method and provide ways for improvements.

\section{Choice of DVB-SH as system of opportunity for positioning}

The DVB-SH is an European standard for broadcasting video content to mobile user using frequencies below $3 \mathrm{GHz}$. DVB-SH uses both geostationary satellite and terrestrial emitters in metropolitan areas in order to provide a continental coverage at lower deployment cost. The standard covers different sizes of signal bandwidth, from 1.7 to $8 \mathrm{MHz}$, to accommodate the different regulatory environments encountered all over the world. The standard is derived from the widely adopted DVB-T (Digital Video Broadcast Terrestrial) standard for fixed digital TV, and from the DVB-H (Digital Video Broadcast - Handheld) standard for mobile digital TV.

Since 2008, two standard documents are available [9] [10], thus finalising all aspects of system definition, and opening the way to the commercial development of receiver chips.

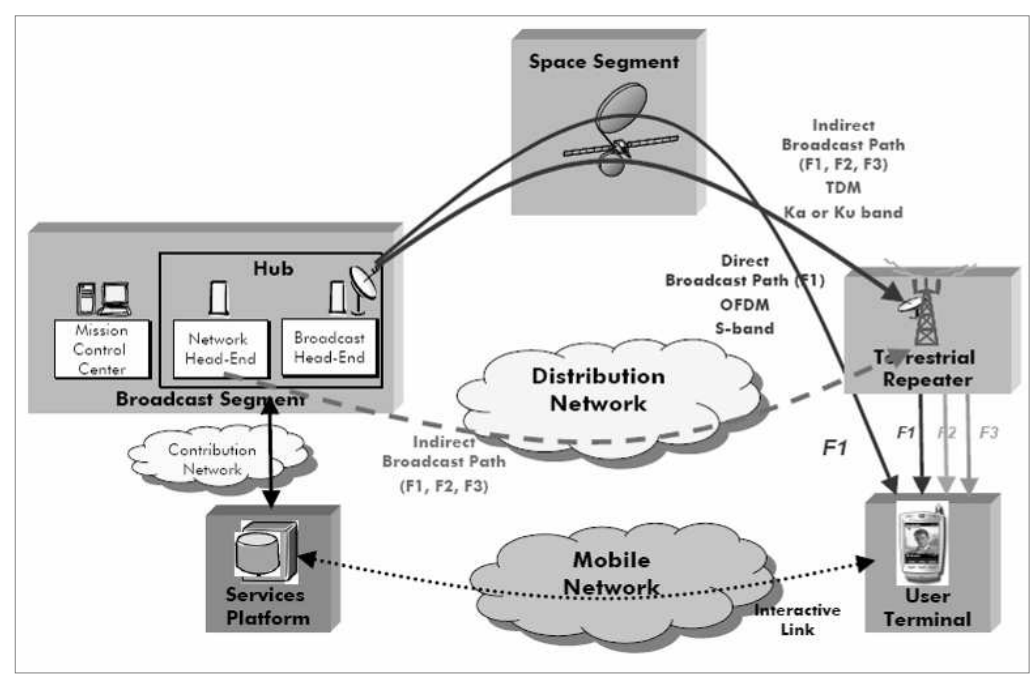

Figure 1 - System architecture of a DVB-SH system [11]

Figure 1 illustrates the architecture of a DVB-SH system. The system comprises:

- A service platform, which performs content adaptation and aggregates TV programs and rich multimedia services into IP service streams.

- A broadcast segment, which maps the IP service streams onto the satellite and terrestrial repeater radio resources

- A dedicated GEO satellite (e.g. W2A, $10^{\circ} \mathrm{E}$, operated by Solaris Mobile Ltd, a joint venture between Eutelsat and SES Astra) that amplifies and convert the satellite radio signals from $\mathrm{Ka} / \mathrm{Ku}$ band to S-band. These signals are transmitted directly to the terminals and provide a cost-effective continental coverage. 
- A network of terrestrial repeaters, which receives service bundles from the broadcast segment either via satellite backhaul or a terrestrial network and converts them onto S-band for delivery to the terminals in zones where the satellite coverage is degraded, such as metropolitan areas. The terrestrial repeaters will also be able to broadcast additional local content, compared to the satellite stream.

- User terminals, which can decode the DVB-SH signals, and which are also likely to be compatible with $3 \mathrm{G}$ networks, thanks to the proximity between the frequency bands used by DVB-SH $(2.2 \mathrm{GHz})$ and $3 \mathrm{G}$ networks $(1.8$ and $2.1 \mathrm{GHz})$.

A typical system capacity is 9 Mbps (2.3 Mbps transferred by a satellite link and 7 Mbps transferred by terrestrial links) [12]. The $2.3 \mathrm{Mbps}$ would have a large coverage (1 country or "language zone") and corresponds to $9 \mathrm{TV}$ programs encoded at $256 \mathrm{kbps}$, which is adapted to typical handheld screen size and resolution.

At the signal level, the DVB-SH comprises two modes of transmission:

- An Orthogonal Frequency Division Multiplexing (OFDM) mode based on DVB-T standard with enhancements. This mode can be used on both the satellite and terrestrial paths; the two signals are combined in the receiver to strengthen the reception in a SFN configuration.

- A Time Division Multiplexing (TDM) mode partly derived from DVB-S2 standard, in order to optimize transmission through satellite towards mobile terminals. This mode is used on the satellite path only. The system supports code diversity recombination between satellite TDM and terrestrial OFDM modes so as to increase the robustness of the transmission in relevant areas (mainly suburban).

The preferred mode of transmission is the first one, and this is the one that is considered in this study. Its main advantage is a much simpler terminal, where only one demodulator is used to receive both the satellite and terrestrial signals.

The choice of DVB-SH as System of Opportunity for positioning can be summarized by the following reasons:

- Synchronized network of emitters thanks to the use of SFN. This enables timing-based pseudorange measurements without the need of costly reference stations.

- Coverage in urban centers and indoor targeting mass market audience (handheld and in-vehicle terminals) thanks to a dense network of emitters composed of a geostationary satellite for continent-wide rural or sub-urban areas, completed by terrestrial gap-fillers in urban areas.

- Convergence with other telecommunication systems at the receiver design level thanks to the carrier frequency around $2 \mathrm{GHz}$, close to other telecommunication bands (e.g. 3G band at 2.1 $\mathrm{GHz}, \mathrm{WiFi}$ band at $2.45 \mathrm{GHz}$ ). Indeed, such "convergence receiver" could share the RF front-end and/or some signal processing functions between both services.

- Wideband signals. Signal bandwidth up to $8 \mathrm{MHz}$ are foreseen, which should be beneficial for positioning accuracy and multipath mitigation.

- OFDM modulation is used as air interface. This modulation is used in many new or upcoming telecommunication standards. There is a strong chance of reuse of this work in other OFDM-using standards.

\section{Overall positioning strategy}

The positioning strategy presented in this paper uses the conventional tri-lateration technique, similar to GNSS systems. The pseudo-ranges between the receiver and different synchronized DVB-SH emitters are 
estimated through signal processing techniques. Then, by knowing the emitters' position, it is possible to solve the positioning problem with a sufficient number of pseudo-ranges.

The overall positioning strategy is explained in Figure 2.

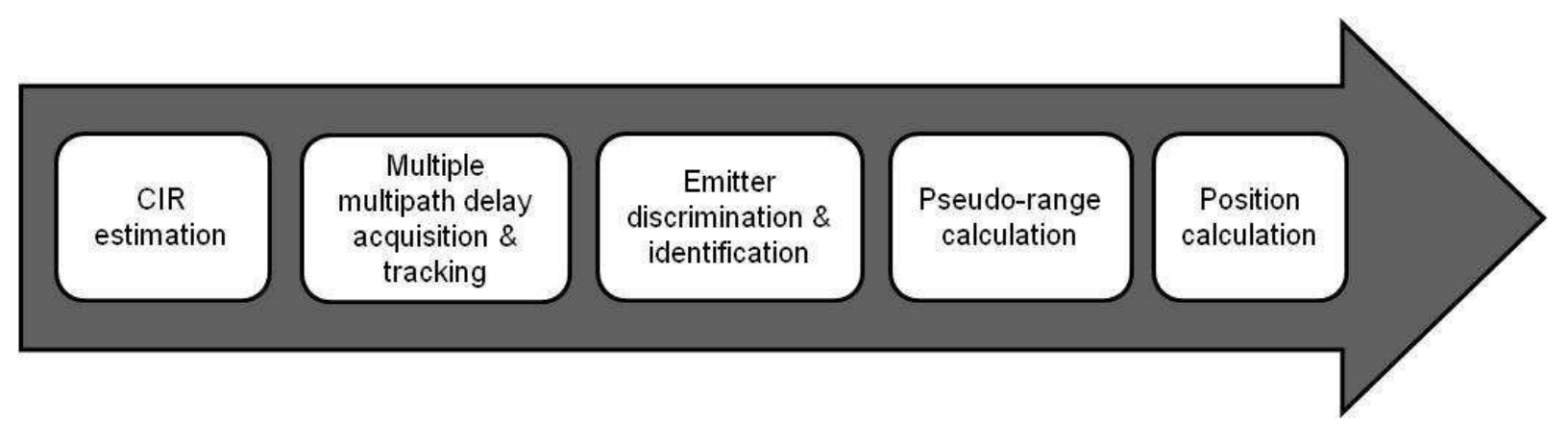

Figure 2 - Positioning strategy using a DVB-SH system

When using a DVB-SH-based system, 2 major issues arise.

- The first one is a system-level issue: as the emitters are working in SFN, it is difficult to identify the transmitter of a given received signal. This issue requires a modification of the system deployment described in the next section.

- The second issue is at the signal processing level. The pseudo-range measurement is challenged by the urban propagation channel. Indeed, the environment between the emitters and the receiver will create multipath and may block the line-of-sight signal. In order to mitigate the effects of this multipath channel, the receiver will aim at estimating the delay of all received signal replicas in the channel impulse response (CIR), and chose the earliest one for the pseudo-range calculation for each emitter. Therefore, at any instant, the receiver may be tracking simultaneously several delays. The different signal processing steps are the main focus of this article and will be described later in this article.

\section{OFDM Signal description}

The DVB-SH standard is described extensively in the [9] and [10].

\section{OFDM modulation principle}

The OFDM modulation [13] consists in transmitting simultaneously several data symbols over several orthogonal sub-carriers. The sub-carrier bandwidth is chosen to be inferior to the channel's coherence bandwidth. Therefore, each sub-carrier is affected by a flat-fading distortion, which can be easily corrected using simple channel estimation techniques. A high data throughput can be obtained by using a large number of sub-carriers, typically several thousands. The digital implementation of this modulation is achieved by the efficient FFT algorithm, allowing for a large number of sub-carriers.

Additionally, a guard interval is inserted between successive OFDM symbols in order to avoid InterSymbol Interference (ISI). This guard interval is used to transmit an exact replica of the end of the OFDM symbol, called Cyclic Prefix (CP). The use of the CP allows for low timing synchronization requirement, since a shift in the FFT window start will only result in a phase shift of each sub-carrier, easily corrected during the channel equalization process. The length of the $\mathrm{CP}$ shall be chosen so as to be longer than the delay of the last expected multipath in order to avoid ISI.

In DVB-SH, the OFDM signal waveform is defined by 3 parameters:

- $\quad \boldsymbol{N}_{\boldsymbol{F F T}}$ - the size of the FFT used for modulation and demodulation. $N_{F F T}$ can take the following values $\{1024,2048,4096,8192\}$. 
- $\quad \boldsymbol{C P}$ - the size of the so-called Cyclic Prefix, which is used as guard interval to avoid inter-symbol interferences due to the delay spread of the signal created by a multipath environment. $C P$ can take the following values $\{1 / 4,1 / 8,1 / 16,1 / 32\}$

- $\boldsymbol{B}$ - the bandwidth of the transmission. $B$ can take the following values $\{1.7,5,6,7,8\} \mathrm{MHz}$.

In this study, only one set of OFDM parameters is used: $N_{F F T}=2048, C P=1 / 4$ and $B=5 \mathrm{MHz}$.

\section{Overall signal illustration}

In an OFDM transmission, symbols are transmitted in parallel over $N_{F F T}$ sub-carriers. There are different types of symbols:

- Null symbols, which are located on sub-carriers on the edge of the transmission spectrum and have a zero value. They act as guard bands, used to limit the out-of-band emissions of the OFDM signal. Their number is determined by the FFT size $N_{F F T}$;

- Data symbols, which are modulated thanks to a Quadrature Amplitude Modulation (QAM), and contain the data payload to be transmitted (the DTV signal). QPSK, 16-QAM and non-uniform 16-QAM mapping are supported by the DVB-SH standard;

- Transmission Parameter Signaling (TPS) symbols, carrying information about transmission parameters, e.g. channel coding and modulation;

- Pilot symbols, which are modulated by a BPSK pseudo-random sequence. Their amplitude is boosted by a factor $4 / 3$ compared to the data or TPS symbols. There are 2 kinds of pilot symbols: continual pilots and scattered pilots, located as shown of Figure 3. Pilots are mainly used for synchronization and channel equalization.

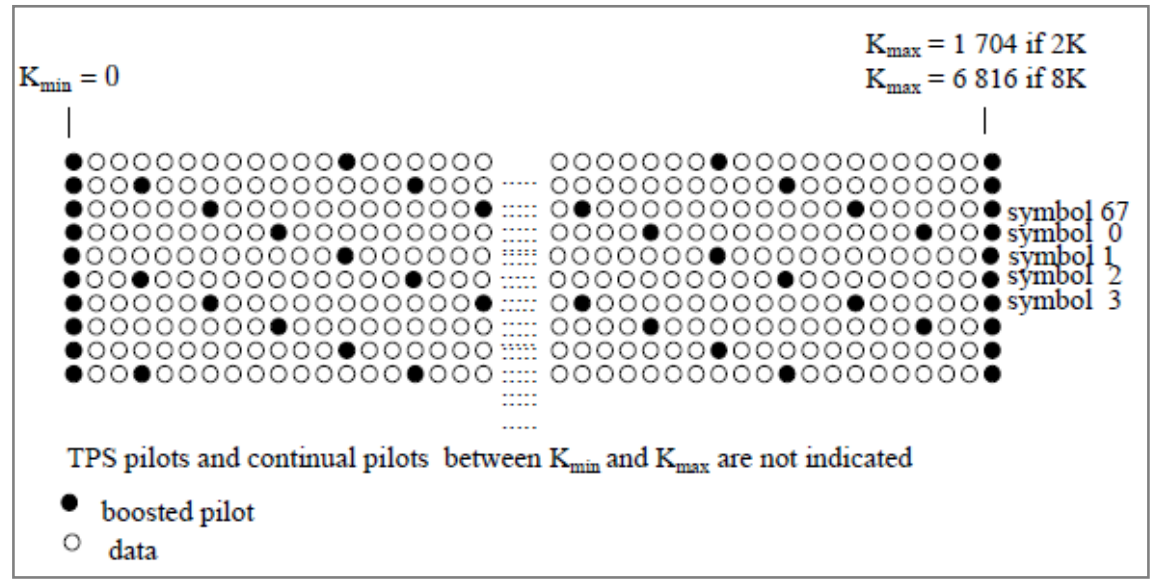

Figure 3 - Pilot location [9]

In the case of the DVB-SH signal investigated in this paper, the 2048 symbols (carried over the same number of sub-carriers) are divided between:

- $\quad K=1705$ useful sub-carriers

○ 45 continual pilot symbols

- 142 scattered pilot symbols (a number of scattered and continual pilots are on the same sub-carrier, thus reducing this number to 131 distinct scattered pilots)

○ 17 Transmission Parameter Signalling symbols, which carry information about the signal parameters

- 1512 data symbols, modulated by 16-QAM 
- 343 null sub-carriers, which are used as guard bands on the edges of the transmission spectrum.

\section{Model of the baseband OFDM transmission chain}

For the rest of this study, OFDM signals are modeled according to the block diagram shown in Figure 4.

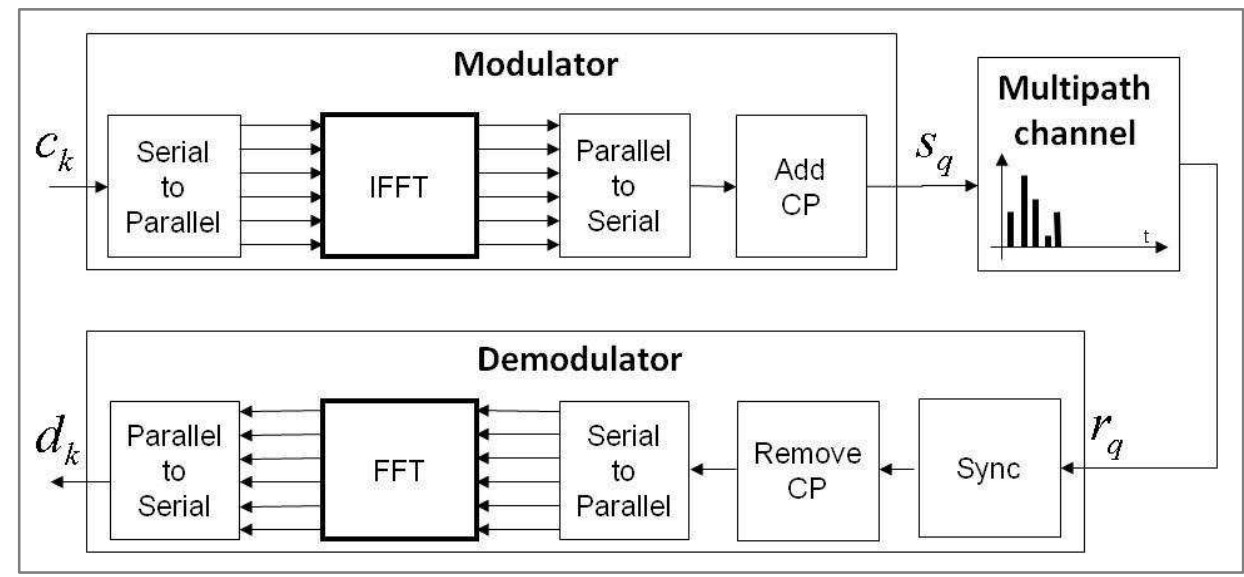

Figure 4 - Model of the baseband OFDM transmission chain

\section{Expression of the demodulated signal model}

It is not necessary to detail mathematically all the intermediary steps of the signal emission and transmission shown in Figure 4. Only the expression of the received, demodulated signal at the output of the transmission chain is of interest. The other previous steps will be briefly explained.

The first operation done by the receiver is the synchronization, both for timing and frequency. Timing synchronization consists in placing the demodulator's FFT window at the correct time, ideally towards the end of the CP near the start of the useful part of the OFDM symbol. Frequency synchronization consists in removing the residual carrier frequency offset which may subsist after carrier removal.

This synchronization step is usually done using the method detailed in [14]. This method consists in using the correlation of 2 slices of the received signal separated by the duration of the useful OFDM symbol length. This correlation should have a peak when the 2 slices are the CP and the end of the OFDM symbol. This means that the receiver knows the location of the beginning of the OFDM symbol. The van de Beek algorithm provides a fine frequency synchronization and a coarse timing synchronization. Therefore, there is no residual carrier frequency offset, but a remaining timing offset, which is assumed to be small enough so as not to create ISI.

After this synchronization step and the FFT demodulation operation, the expression of the demodulated symbols is:

$$
d_{l, k}=e^{j \varphi_{0}} \cdot c_{l, k} \cdot H_{l, k} \cdot e^{-j 2 \pi \frac{k \Delta \tau}{N_{F F T}}}
$$

where $d_{l, k}$ is the demodulated symbol on the $k$-th sub-carrier of the $l$-th OFDM symbol

$c_{l, k}$ is the sent symbol on the $k$-th sub-carrier of the $l$-th OFDM symbol

$\Delta \tau$ is the remaining timing offset after the van de Beek synchronization

$H_{l, k}$ is the channel frequency response on the $k$-th sub-carrier at the time of reception of the $l$-th OFDM symbol

$\varphi_{0}$ is a random initial phase

The demodulated symbol is affected by 
- a complex distortion $H_{l, k}$ depending on the sub-carrier index $k$, which is created by the propagation channel;

- a phase shift depending on a random term $\varphi_{0}$, the sub-carrier index $k$ and the residual timing offset $\Delta \tau$.

Both these impairments can be corrected during the channel equalization step. This equalization consists in using pilot symbols in order to estimate the channel frequency response and the phase shift, and then dividing the demodulated symbol by this estimate in order to obtain the initially sent symbol $c_{l, k}$.

\section{SFN channel impulse response modeling and associated issues}

\section{SFN principle}

In a DVB-SH system, the emitters may work in an SFN. An SFN is a network of emitters that broadcast:

- the same signal

- on the same carrier frequency

- in a synchronized way.

Therefore, a receiver in an SFN will receive several delayed replicas of the same signal. As long as the receiver is able to cope with those multipaths (through equalization techniques for example) this will increase the power of the useful signal. If the multipaths are too intense (for example the delays of the replicas are outside the equalizer range of the receiver), those replicas will create interferences. Therefore, the SFN deployment shall take into account the receiver's equalizer's performances in order to avoid selfinterferences within the system. [15] describes the requirements and benefits of the SFN in a broadcast.

\section{Urban SFN issues for positioning}

The SFN principle was proposed with the goal of optimizing the telecommunication service. However, the use of this network configuration raises 2 major issues for positioning:

- Delay overlap: the signal replicas coming from different emitters may arrive simultaneously, making emitter discrimination or identification difficult. This is an issue for positioning, since the receiver has to know the emitter of origin of the signal it tracks in order to be able to compute its position.

- Near-Far Effect: the relative distance between the receiver and different emitters will greatly vary during the receiver's motion. This may create inter-emitter interferences, ie a close emitter would mask the signals arriving from more remote emitters. This causes an issue for positioning, since the receiver aims at tracking the signals coming from several different emitters, even remote ones.

In addition to the particular emitter network configuration used in DVB-SH systems, the urban propagation channel also presents other issues for positioning. Among others extensively described in [16], the particularities of the urban propagation channel are:

- The presence of multipaths, which consists in delayed replicas of the emitted signal created through interactions (reflections, diffraction, etc.) with the environment between the emitter and the receiver. The consequences of this multipath propagation is fast and strong fading of the received signal power for close multipath, the reception of multiple replicas of the signal for large multipath, and large average power decay vs distance (aka pathloss) created by the overall environment. 
- Masking / blocking of the line-of-sight (LOS) signal by obstacles. This means that other multipath may be received with a stronger power.

Both these phenomena are challenging for a positioning system since the receiver aims at using a measurement of the time of arrival of the LOS signal in order to estimate the distance to one emitter. If a multipath is used instead of the LOS signal, the pseudo-range will be affected by a bias, thus degrading the final user position.

Also, aside from pure propagation-related issues, some system-related issue may also affect the positioning technique:

- Synchronization of the SFN: the synchronization between the emitters may not be perfect, thus resulting in errors on each pseudo-range. However, it can be safely assumed that the current stateof-the art emitter synchronization achievable with a GPS receiver is of the order of $50 \mathrm{~ns}$ delay jitter - resulting in a $15 \mathrm{~m}$ error jitter on the pseudo-range - and that this performance will go on being improved in the future. In this study, it was assumed that a perfect synchronization was achieved.

- Number of visible emitters: in order to be able to compute a 2D position using a tri-lateration technique, a minimum of 3 emitters is required. The network deployment of DVB-SH systems is done in order to provide a minimum of one visible emitter for the service coverage. However, the visibility criteria for telecommunication system planning is based on the Signal-to-Noise Ratio being above the data demodulation threshold. This demodulation threshold is much higher than the tracking threshold required in order to obtain a pseudo-range by the proposed technique. Therefore, it has been demonstrated that a sufficient number of emitters will be available to compute a position in such network deployment [18].

\section{Proposition of SFN modification for positioning}

One solution proposed by the authors in [17] is to introduce artificial delay between the emission of different emitters, in order to avoid the delay overlap. This may also solve the near-far effect issue, since once the signal replicas arrive with different delays, they will less interfere with each other.

The introduction of artificial delay will not degrade the telecommunication service, as long as the signal replicas arrive within the equalization range of the receiver, which is also called the CP for OFDM signals, as used in the DVB-SH standard. A detailed study of the compatibility between the DVB-SH signal parameters and this system modification can be found in [18].

The introduction of artificial delay is already planned in the DVB-SH standard, in order to facilitate network deployment. Therefore, this proposition shall have only a minor impact on the system deployment.

\section{Illustration of SFN CIR time series}

In order to account for all the propagation channel specificities created by the urban environment and the SFN configuration, results from a channel sounding measurement campaign have been used. In the frame of the S-DMB project (a DVB-SH pre-standardization project), CNES has realized a measurement campaign in urban and sub-urban environments that was used to estimate the propagation channel during the experiment [19]. The measurements used in this section were made in Auch, France ( 20,000 inhabitants) with 2 terrestrial emitters and one helicopter (simulating a satellite) simulating a SFN, and a moving van carrying a channel sounder. The transmission was done with a carrier frequency at $2.2 \mathrm{GHz}$ in order to characterize the DVB-SH conditions. The path followed by the van during the measurement is shown in Figure 5 and its speed varied between 0 and $40 \mathrm{~km} / \mathrm{h}$. 


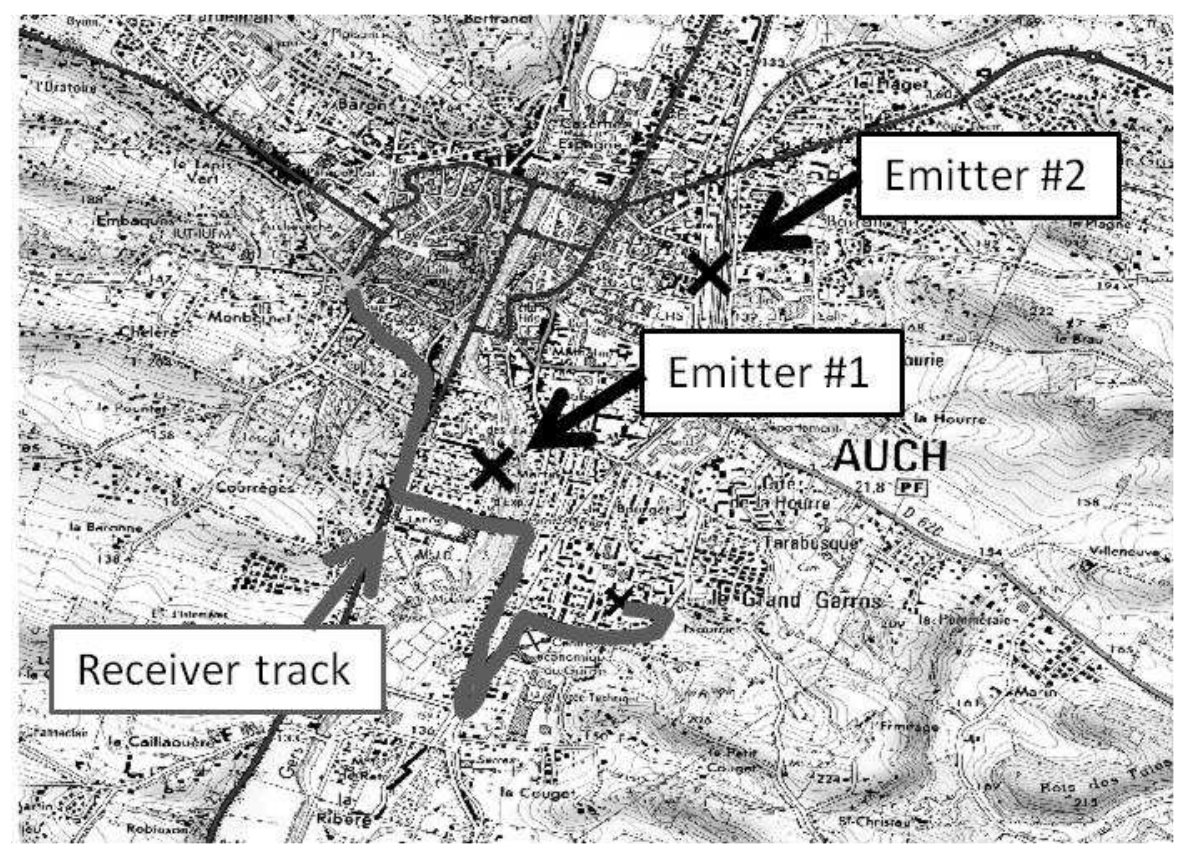

Figure 5 - Path followed by the receiver. The 2 terrestrial emitters are located at the crosses.

The measurements consist in the pathloss and the delay of 6 taps for the terrestrial emitters and only the pathloss of a single tap for the satellite emitter. Along with the position and the speed of the van, these parameters are used in order to generate the SFN CIR time series used in the simulation.

Figure 6 shows the power of the received signal replicas from the different emitters along the path. An EIRP of $53.2 \mathrm{dBm}$ was used for the terrestrial emitters, as used in a typical DVB-SH link budget presented in [12], while the satellite EIRP is $101.3 \mathrm{~dB}$ (also according to [12]), and free space loss is added, with the satellite position assumed at a longitude of $10^{\circ} \mathrm{E}$ on the geostationary orbit.

Figure 6 was plotted using a running average over 4 seconds, thus filtering the fast fading. In the actual measurement without filtering, deep fading of several tens of $\mathrm{dB}$ occurs on every replica with fast variations, according to the speed of the receiver. The remaining variations are due to the distance variation between the receiver and the emitters, and also to the changing shadowing conditions. The NearFar Effect is clearly illustrated with power difference up to $40 \mathrm{~dB}$ between the different emitters. 


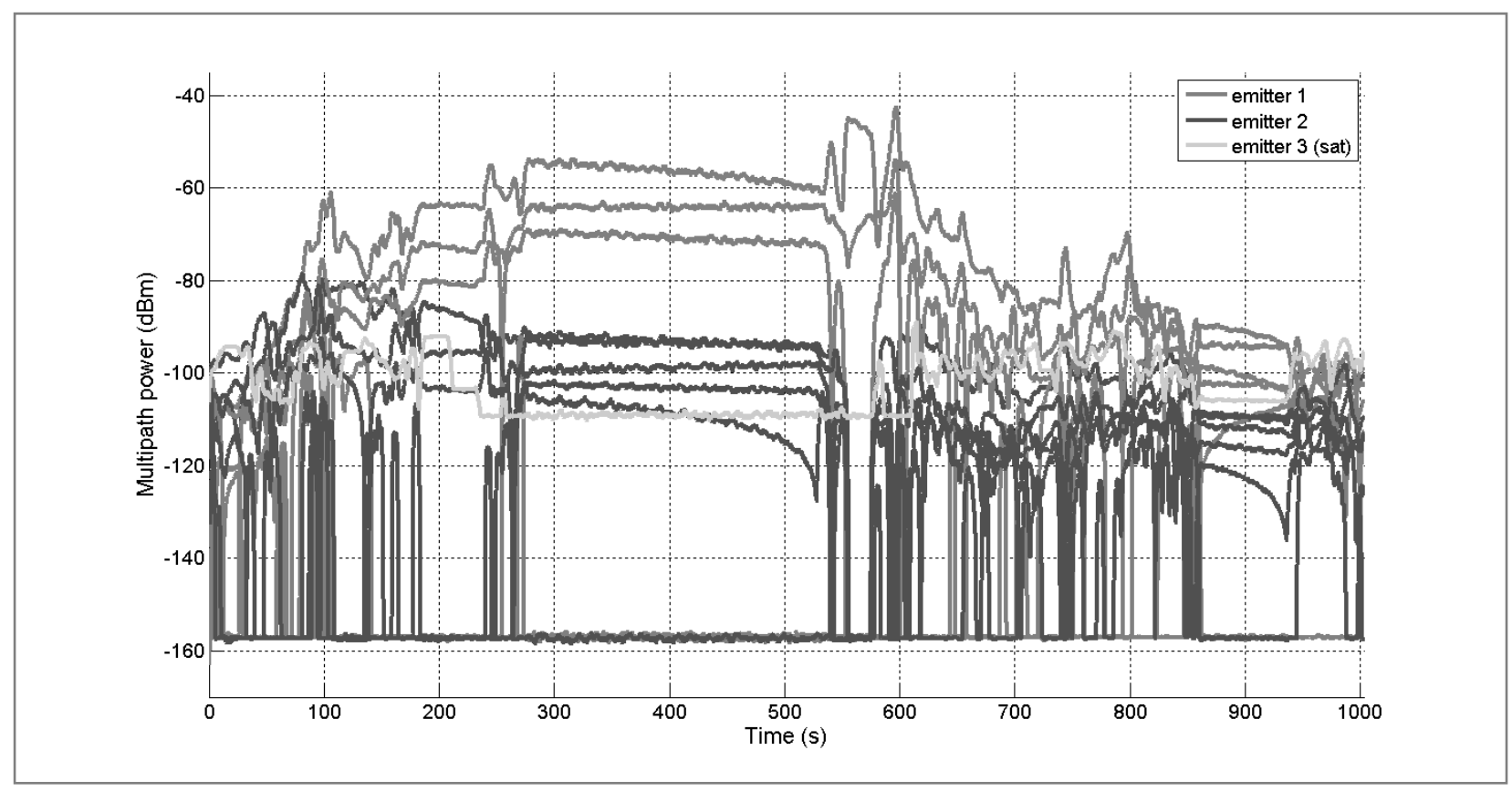

Figure 6 - Time series of the power of the signal replicas coming from the SFN averaged over 4 seconds

Figure 7 shows the delay of the received signal replicas after the introduction of artificial delay: a $6 \mu \mathrm{s}$ delay was introduced for emitter \#1 and a $12 \mu$ s delay was introduced for emitter \#2. This artificial delay introduction solves the delay overlap issue. Multipaths whose power is below $-120 \mathrm{dBm}$ have been put in thin lines, so that we observe only the most significant multipaths and the fading affecting them.

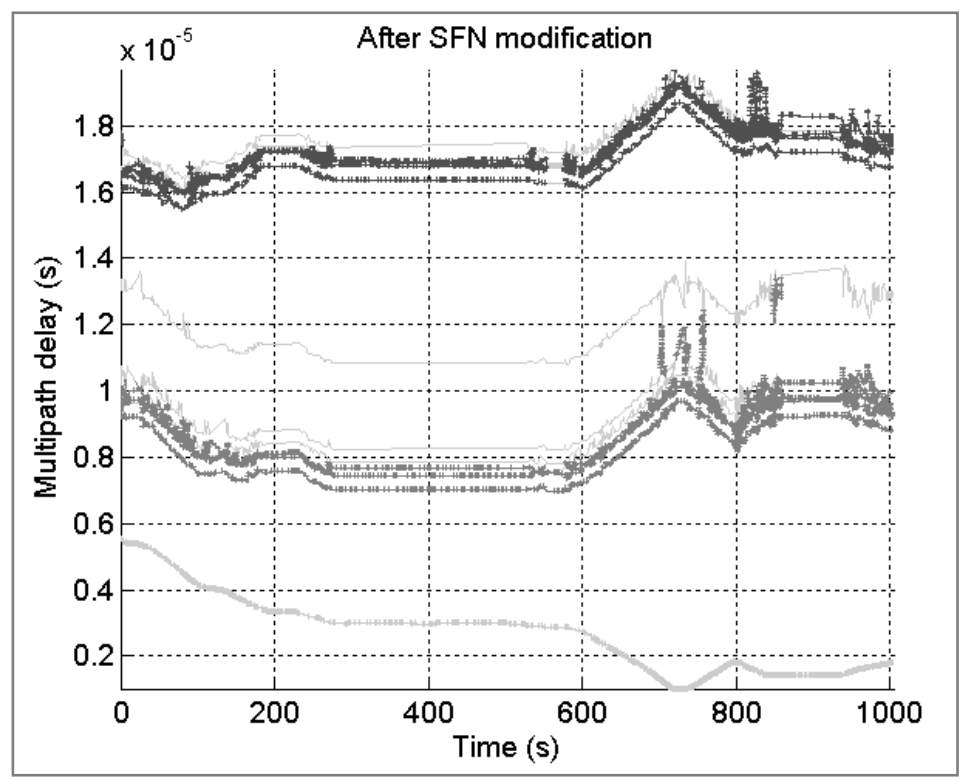

Figure 7 - Multipath delay in the SFN after artificial delay introduction

\section{Pseudo-range estimation technique from an OFDM signal \\ CIR estimation by correlation}

CIR estimation is done by correlating the received signal with a local replica of the expected signal. This local replica is composed of the pilot symbols on the pilot sub-carriers and null symbols elsewhere. The correlation process is illustrated by the following block diagram. 


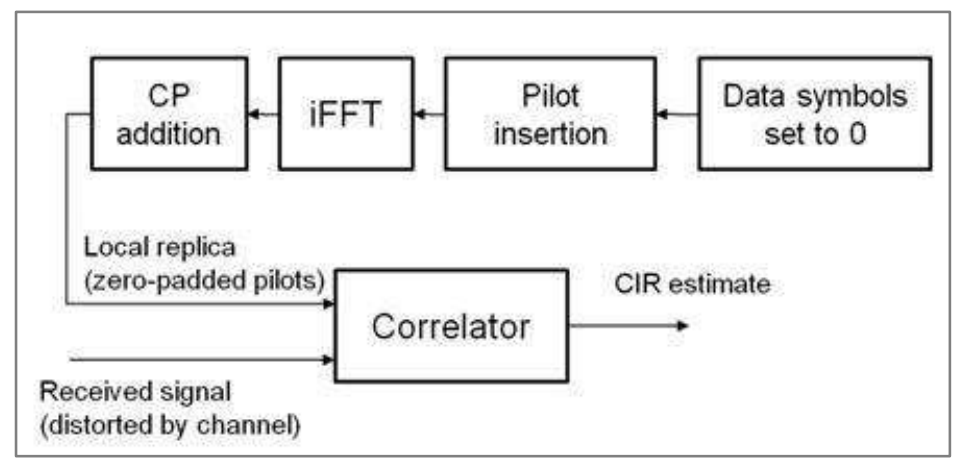

Figure 8 - Correlation operation done to estimate the CIR

The received signal expression is described in Eq. 1. The local replica is composed of the scattered pilots only, meaning that all other sub-carriers are set to a null value. The correlation output general expression is:

$$
R_{l}(\Delta t)=\frac{1}{N_{p}} \sum_{q \in \mathcal{P}} d_{l, q} \cdot p_{l, q}^{*}(\Delta t)
$$

Eq. 2

where $l$ corresponds to the time of reception of the $l$-th OFDM symbol;

$q$ corresponds to the sub-carrier index;

$d_{l, q}$ is the expression of the received signal after Van de Beek synchronization and FFT demodulation. See Eq. 1;

$p_{l, q}$ is the expression of the local replica, containing scattered pilot only symbols. $p_{l, q}=c_{l, q}$ if $q \in \mathcal{P}$ and $p_{l, q}=0$ otherwise;

$\mathcal{P}$ is the sub-carrier index set. In the DVB-SH standard, there is one scattered pilot every 12 subcarrier, as shown in Figure 3;

$N_{p}$ is the number of scattered pilots in one OFDM symbol;

$\Delta t$ is the delay offset between the local signal replica and the received signal.

After a few manipulations, the correlation output expression becomes [20]

$$
R_{l}(\Delta t)=\frac{\sigma_{p}^{2}}{N_{p}} \cdot \sum_{n=0}^{N_{M P}-1} \alpha_{l, n}(\Delta t) \cdot \frac{\left.\sin \left(\pi \beta \cdot\left(\tau_{n}+\Delta \tau-\Delta t\right)\right)\right)}{\left.\sin \left(\frac{\pi \beta}{N_{p}} \cdot\left(\tau_{n}+\Delta \tau-\Delta t\right)\right)\right)}
$$

where $\sigma_{p}^{2}$ is the variance of the pilot symbols;

$N_{M P}$ is the number of received signal replicas;

$a_{l, n}$ is the amplitude of the $n$-th multipath present at the time of reception of the $l$-th OFDM symbol;

$\tau_{l, n}$ is the delay normalised by the sample duration $T_{\text {samp }}$ of the $n$-th multipath present at the time of reception of the $l$-th OFDM symbol;

$\alpha_{l, n}(\Delta t)=a_{l, n} \cdot e^{-\frac{j \pi\left(\tau_{n}+\Delta \tau-\Delta t\right)}{N_{F F T}} \cdot\left(2 \cdot N_{0}+P .\left(N_{p}-1\right)\right)}$ is a complex factor accounting for the multipath complex amplitude and a phase term depending on many parameters;

$N_{0}$ corresponds to the index of the first non-null sub-carrier. This value depends on the OFDM symbol index $l$ and on the FFT size $N_{F F T}$;

$P$ is the scattered pilot period. It is equal to 12 in the DVB-SH standard;

$\beta=\frac{P N_{p}}{N_{F F T}}=0.832$. It is a constant in the DVB-SH standard. 
The correlation expression indicates that the shape of the correlation peak can be approximated by a sinc function for small delays (Figure 9). The amplitude of the peak corresponds to the boosted amplitude of the sent symbols $\left(\sigma_{p}^{2}=4 / 3\right)$.

Put in other words, the correlation operation provides an estimation of the CIR convoluted by the correlation peak shape function. Its amplitude can therefore be used as an estimator of the CIR.

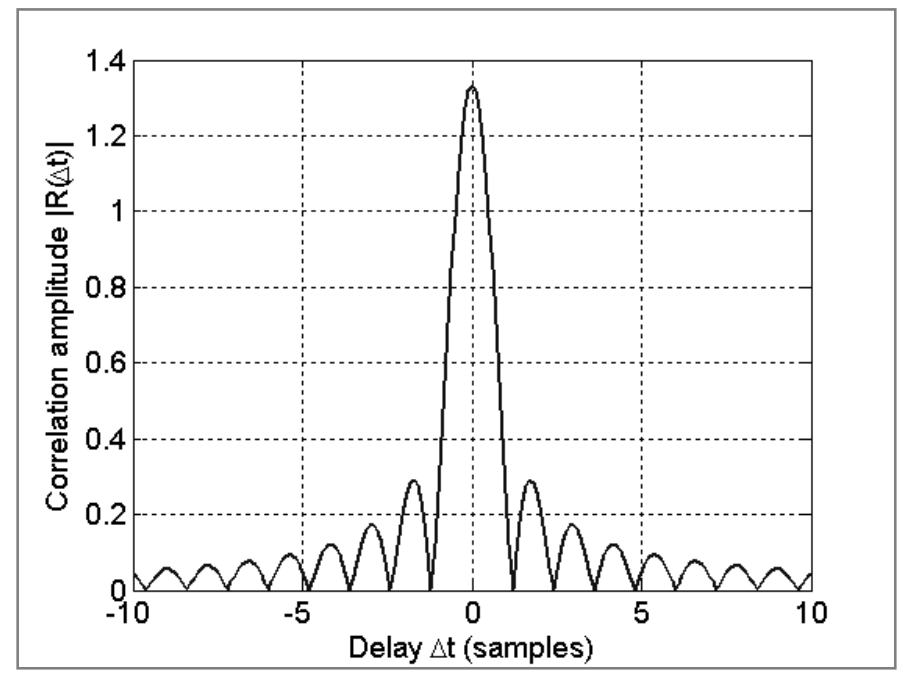

Figure 9 - Correlation amplitude for a single-tap channel.

The correlation operation is applied to a DVB-SH signal filtered by the SFN CIR measurement previously presented. The result is shown in Figure 10. The delay evolution can be observed on the $x-y$ plane, while the color corresponds to the amplitude of the correlation output ("hot" colors correspond to high value of the correlation amplitude).

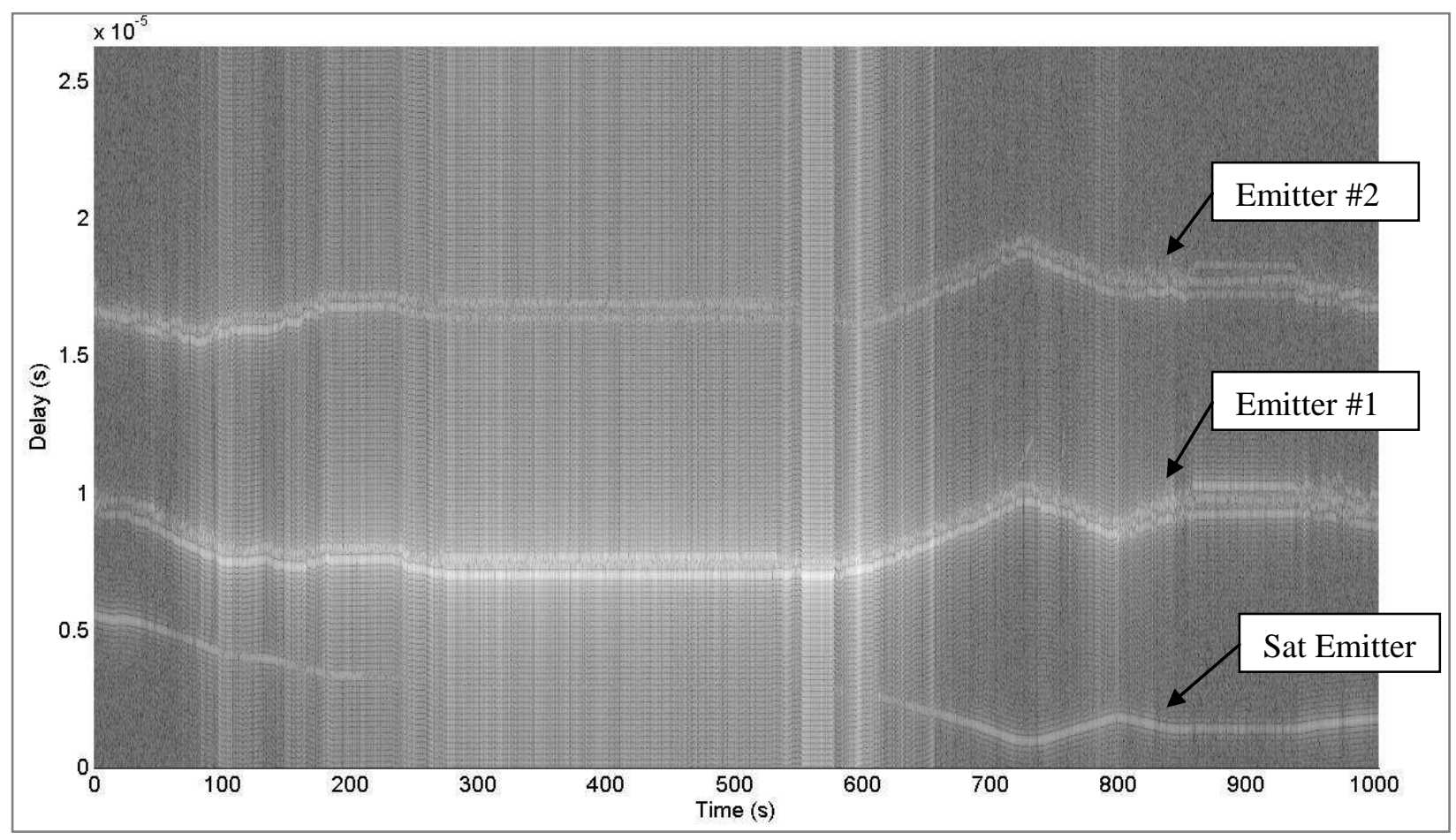

Figure 10 - Correlation output amplitude of a DVB-SH filtered by the measured SFN CIR

The goal of the overall pseudo-range estimation process is to detect and track the peaks in the CIR estimate created by each emitter. Figure 10 shows that even if the delay overlap issue has been solved by the introduction of artificial delays between the 3 emitters, there still exists an influence of one emitter on 
another due to the presence of sidelobes in the correlation peak function. This influence is problematic, notably due to the Near-Far Effect. In this figure, emitter \#1 is received with much higher power than the other emitters. The sidelobes of the correlation peaks corresponding to emitter \#1 are even drowning the correlation peak of the other emitters, making their detection or tracking difficult or even impossible.

In order to mitigate this issue, the use of windowing techniques is proposed.

\section{Use of windowed correlation}

The use of a windowing technique in the correlation is done by weighing the local replica by a windowing function. In this case, Eq. 2 becomes:

$$
R_{l}^{\text {window }}(\Delta t)=\frac{1}{N_{p}} \sum_{q \in \mathcal{P}} c_{l, q} \cdot p_{l, q}^{*}(\Delta t) \cdot w_{\text {window }}(q)
$$

where $w_{\text {window }}(q)$ is the window function.

Two windows have been considered:

- The Hamming window, with the following expression:

$$
w_{\text {Ham }}(q)=0.53836-0.46164 \cos \left(\frac{2 \pi q}{K-1}\right)
$$

- The Blackman-Harris window, with the following expression:

$$
w_{B H}(q)=0.35875-0.48829 \cos \left(\frac{2 \pi q}{K-1}\right)+0.14128 \cos \left(\frac{4 \pi q}{K-1}\right)-0.01168 \cos \left(\frac{6 \pi q}{K-1}\right)
$$

where $K$ is the number of non-null subcarriers.

The case without window corresponds to a rectangular window, where $w(q)=1$.

Figure 11 shows the correlation peak shape for the different windows. Compared to the case without window, the Blackman-Harris window strongly attenuates the sidelobes, but in return, its main lobe is larger and lower. The Hamming window stays in-between.

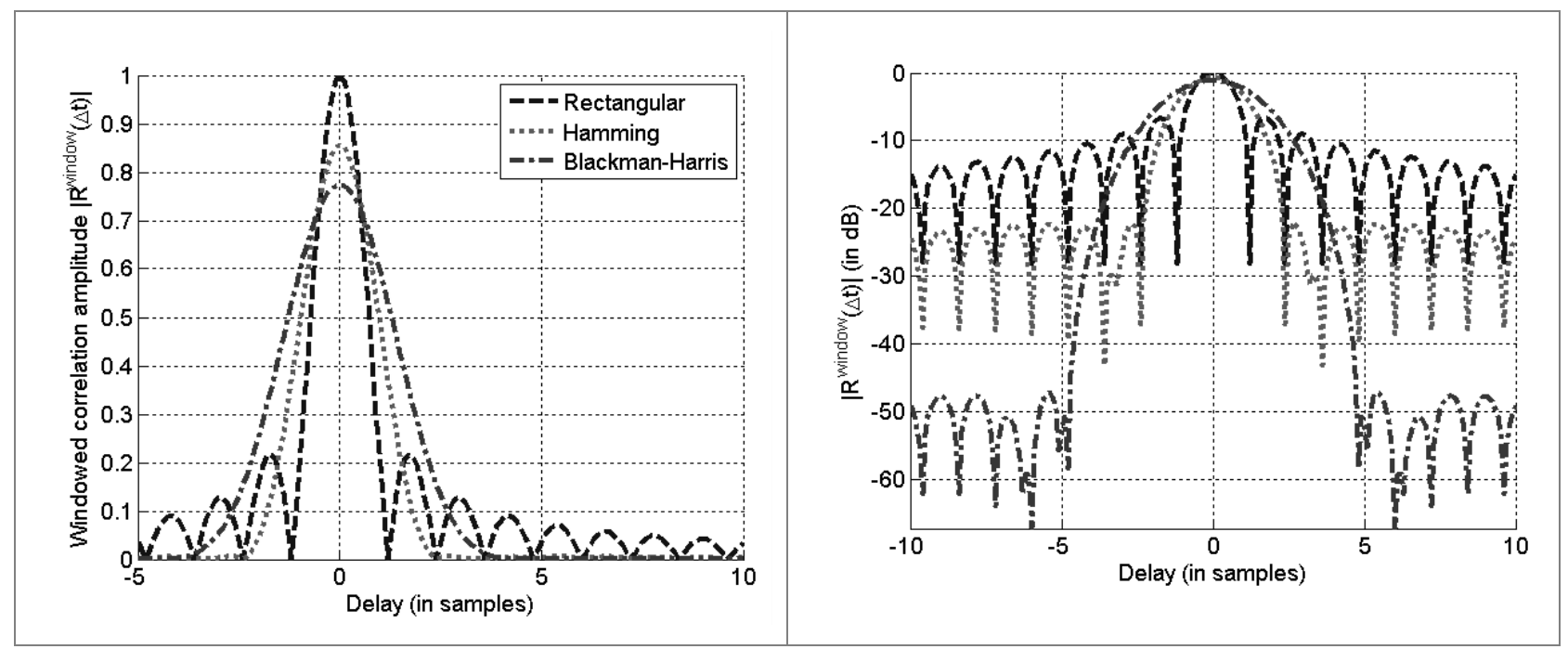

Figure 11 - Correlation peak comparison with windowing techniques

Figure 12 and Figure 13 shows the resulting CIR estimation of the DVB-SH signal filtered by the studied SFN CIR measurement using a windowed correlation. The sidelobes are much lower. In the case of the 
Hamming window (Figure 12), the satellite emitter may still be affected by the sidelobes coming from emitter \#1. In the case of the Blackman-Harris window (Figure 13), the sidelobes are no longer visible.

However, the reduction of the sidelobes is done at the expense of the main lobe sharpness. Therefore, multipath delay extraction using the windowed correlation is expected to yield a lower accuracy due to the main lobe widening.

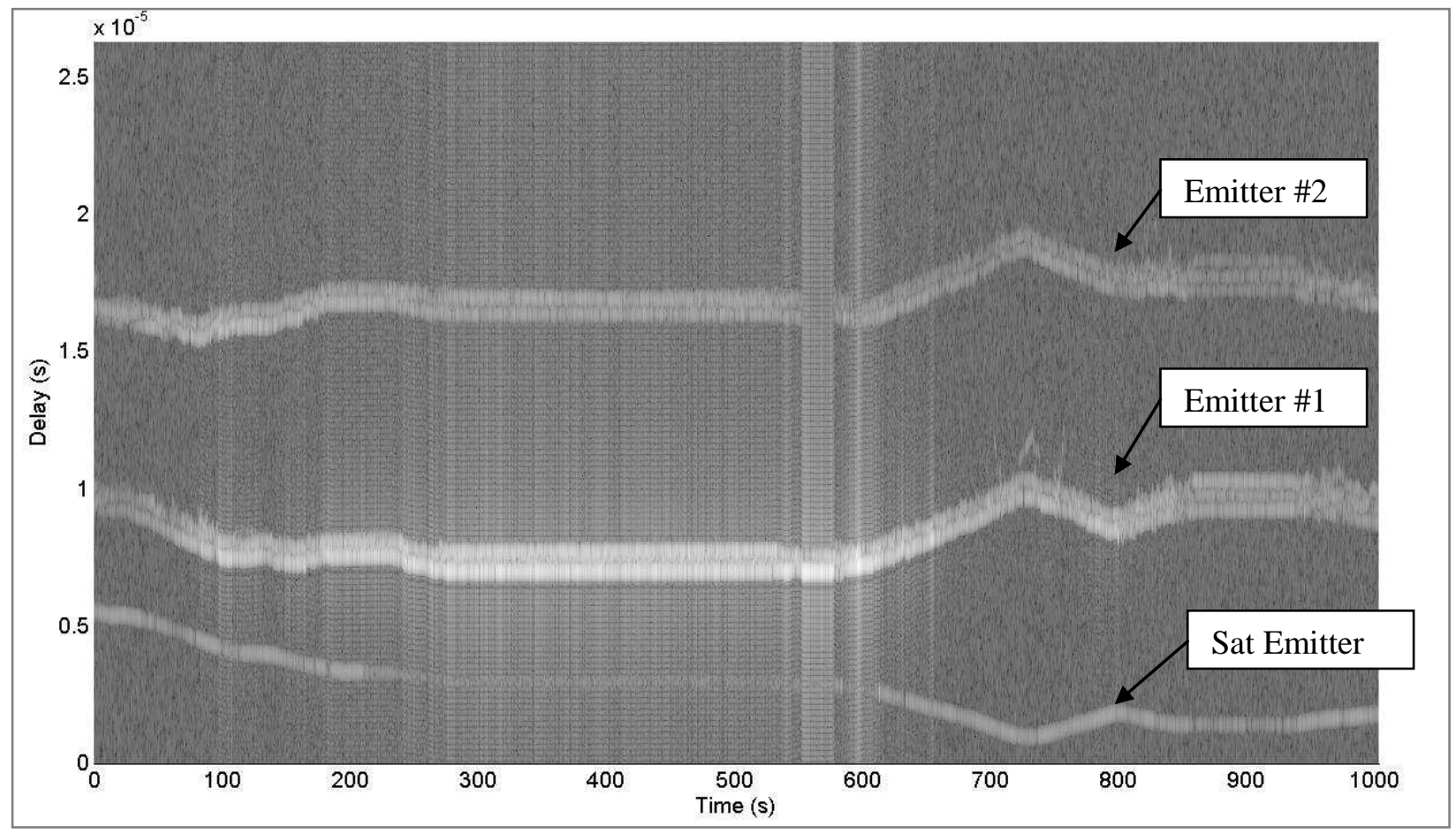

Figure 12 - Correlation output amplitude of a DVB-SH signal filtered by the measured SFN CIR using a Hamming window

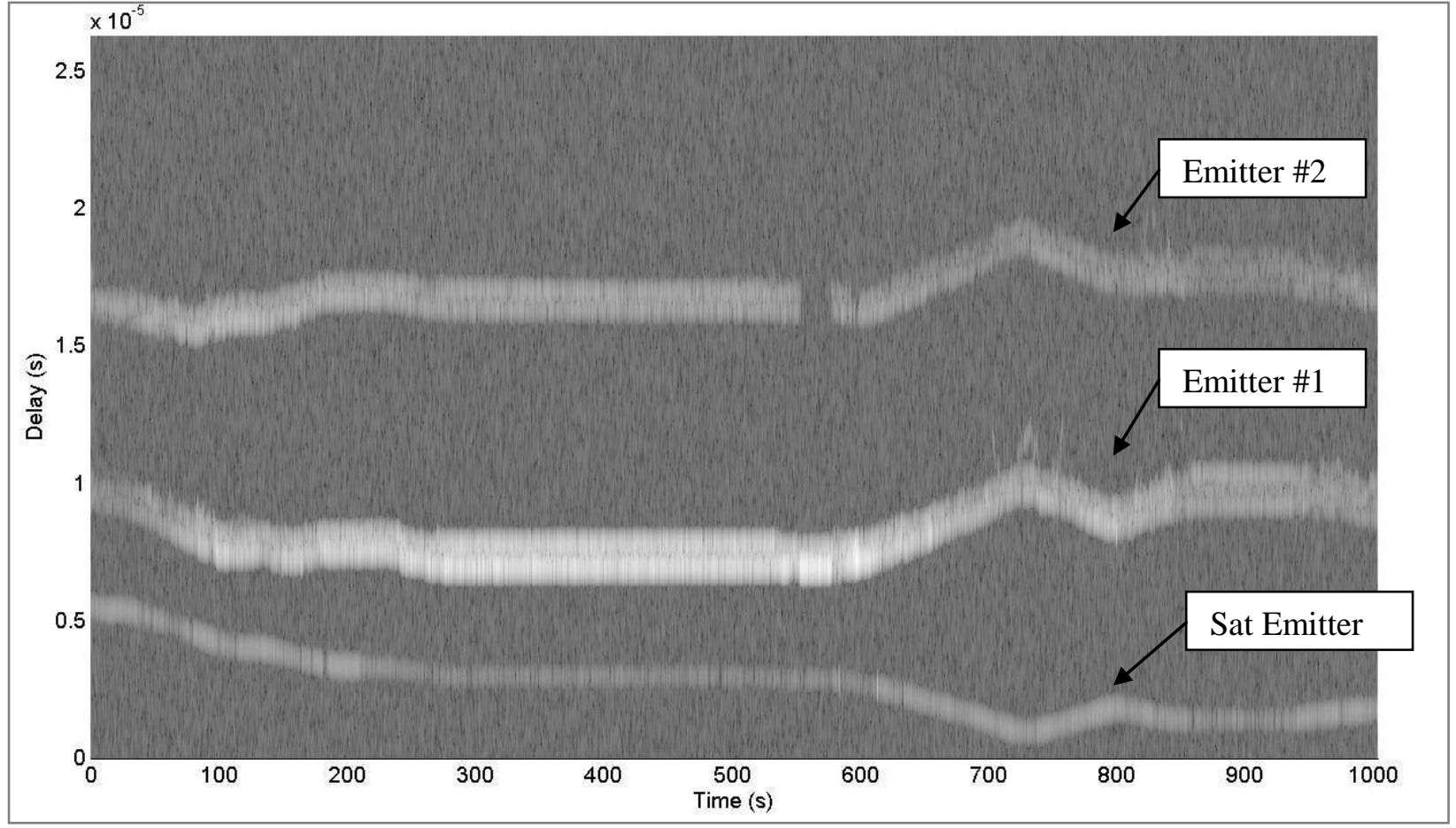

Figure 13 - Correlation output amplitude of a DVB-SH signal filtered by the measured SFN CIR using a Blackman-Harris window 


\section{Multipath delay acquisition by Matching Pursuit}

The correlation function over a wide range of delay provides an estimate of the CIR as seen in the previous section.

The Matching pursuit algorithm [21] can be used to extract the multipath delays from the CIR estimate. This algorithm is well suited to estimate a CIR with sparse multipaths. This is the case in SFN signals, where the signal replicas may be received with significant relative delays.

The goal is to find the family $\rho_{l}, \varphi_{l}$, and $\tau_{l}$ for $l \in\left[1 . . N_{M P}\right]$ that minimizes:

$$
\sum_{k=1}^{K}\left\|\hat{h}(k)-\sum_{l=1}^{N_{M P}} \rho_{l} e^{j \varphi_{l}} f\left(k-\tau_{l}\right)\right\|^{2}
$$

Eq. 5

where $K$ is the length of the CIR estimate vector

$N_{M P}$ is the number of considered multipaths

$\hat{h}(k)$ is the CIR estimate

$f(k)$ is the pulse model of the estimated multipath

Eq. 5 can be rewritten using matrix notation:

$$
\hat{\rho}_{l}, \hat{\varphi}_{l}, \hat{\tau}_{l}=\arg \min _{\hat{\rho}_{l}, \hat{\varphi}_{l}, \hat{\tau}_{l}}\|\hat{\mathbf{h}}-\mathbf{P} \boldsymbol{\beta}\|^{2}
$$

Eq. 6

where $\hat{\mathbf{h}}=\left[\hat{h}_{1}, \hat{h}_{2}, \ldots, \hat{h}_{K}\right]$ is the vector of the CIR estimates

$\mathbf{P}=\left[\mathbf{P}_{1}, \mathbf{P}_{2}, \ldots, \mathbf{P}_{N_{M P}}\right]$ is a KxL matrix containing the pulse samples for each considered multipath

$\mathbf{P}_{l}=\left[f\left(1-\tau_{l}\right), f\left(2-\tau_{l}\right), \ldots, f\left(K-\tau_{l}\right)\right]^{T}$ is the pulse model for the $l$-th delay

$\boldsymbol{\beta}=\left[\rho_{1} e^{j \varphi_{1}}, \rho_{2} e^{j \varphi_{2}}, \ldots, \rho_{N_{M P}} e^{j \varphi_{N_{M P}}}\right]^{\mathrm{T}}$ is the vector of the complex amplitude of the taps

The principle is to find iteratively the columns of $\mathbf{P}$ that best match the CIR estimates (the column index corresponds to the delay of the taps). After each iteration, the CIR vector is corrected by removing the pulse of the previously estimated path and a new delay is estimated. More details can be found in [22]

The Matching Pursuit algorithm is able to provide estimates of the delays of the multipaths present in a CIR for the expected SNR conditions. However, it has 2 inherent drawbacks:

- the algorithm is very cumbersome: it requires a correlation output over a large range of delays and each iteration step requires a large matrix calculation.

- the delay estimates do not use the potentially useful information about the multipath delays estimated at previous instants. This latter drawback is also a strength, since it will permit to obtain delay estimates even when no previous information is available.

For these 2 reasons, the Matching Pursuit algorithm is well adapted for a delay acquisition phase. Multipath delay estimation can then be followed by a delay tracking phase, described in the next section. Note that other methods such as SAGE [23] or ESPRIT [24] could also be used for this acquisition phase.

\section{Multipath delay tracking by Delay-Lock Loop}

The Matching Pursuit algorithm provides a set of delay estimates, which can be used to initiate a tracking phase using multiple Delay-Lock Loop (DLL), one DLL for each delay estimated during the acquisition phase. The DLL will be used to obtain a fine delay estimate, with a much finer resolution than the one obtained previously.

\section{Discriminator's output expression}


The studied DLL [25] uses 3 correlators calculating the correlation function at 3 different instants $(-\delta / 2$, $0,+\delta / 2$ ) and combines them into a particular discriminator, to obtain the updated value of the delay estimation. The parameter $\delta$ is called the correlator spacing and is normalized by the sample duration.

Assuming a single-tap channel and using Eq. 3, with an assumption on a small delay offset between the current estimated delay and the true multipath delay, the correlators' outputs can be written as follows:

$$
\begin{gathered}
R_{l}^{\text {early }}(\varepsilon) \triangleq R_{l}\left(\Delta t-\frac{\delta}{2}\right)=\sigma_{p}^{2} \cdot \alpha_{l} \cdot \operatorname{sinc}\left(\pi \beta \cdot\left(\varepsilon-\frac{\delta}{2}\right)\right) \\
R_{l}^{\text {late }}(\varepsilon) \triangleq R_{l}\left(\Delta t+\frac{\delta}{2}\right)=\sigma_{p}^{2} \cdot \alpha_{l} \cdot \operatorname{sinc}\left(\pi \beta \cdot\left(\varepsilon+\frac{\delta}{2}\right)\right) \\
R_{l}^{\text {prompt }}(\varepsilon) \triangleq R_{l}(\Delta t)=\sigma_{p}^{2} \cdot \alpha_{l} \cdot \operatorname{sinc}(\pi \beta \varepsilon)
\end{gathered}
$$

where $\varepsilon \triangleq \Delta t-\left(\tau_{l}+\Delta \tau\right)$ is the offset between the $l$-th multipath delay and the true multipath delay. We will call $\varepsilon$ the estimation error.

The discriminator used in the proposed DLL is an early minus late power discriminator that can be expressed as follows:

$$
D^{\text {norm }}(\varepsilon)=\frac{1}{K_{\text {norm }}} \cdot \frac{\left|R_{l}^{\text {early }}(\varepsilon)\right|^{2}-\left|R_{l}^{\text {late }}(\varepsilon)\right|^{2}}{\left|R_{l}^{\text {prompt }}(\varepsilon)\right|^{2}}
$$

where $\quad K_{n o r m} \triangleq \frac{1-\frac{\delta}{2} \cdot \pi \cdot \beta \cdot \sin (\pi \beta \delta)-\cos (\pi \beta \delta)}{\pi^{2} \beta^{2} \cdot \frac{\delta^{3}}{16}}$ is a normalization factor [Serant, 2010].

The discriminator's outputs are then fed to a tracking loop, whose parameters are taken from [26].

\section{Discriminator's output variance}

Under thermal noise only hypothesis (no multipath), the variance of the estimated delay can be expressed theoretically [20]:

$$
\operatorname{Var}(\Delta \hat{t})=2 B_{l} T_{\text {symb }} \frac{K_{1}}{S N R}\left(1+\frac{K_{2}}{S N R}\right)
$$

where $B_{l}$ is the DLL equivalent noise loop bandwidth

$T_{\text {symb }}$ is the symbol duration and corresponds to the time interval between 2 delay estimates $S N R=\alpha^{2} / \sigma_{n}^{2}$ is defined as the ratio between the signal power $\alpha^{2}$ and the noise variance $\sigma_{n}^{2}$

$K_{1}=\frac{9}{4} \cdot \frac{\left(1-\operatorname{sinc}\left(\pi \beta^{\prime} \delta\right)\right) \operatorname{sinc}\left(\frac{\pi \beta^{\prime} \delta}{2}\right)^{2}}{N_{p} \cdot K_{\text {norm }}^{2}}$

$K_{2}=\frac{9}{32} \cdot \frac{1+\sin c\left(\pi \beta^{\prime} \delta\right)}{N_{p} \operatorname{sinc}\left(\frac{\pi \beta^{\prime} \delta}{2}\right)}$

$\beta^{\prime}=\beta / K_{\text {width }}^{\text {window }}$

$K_{\text {width }}^{\text {window }}$ is a factor used to account for the window's main lobe widening. For the Hamming window, $K_{\text {width }}^{\text {Ham }}=1.5$ and for the Blackman-Harris window $K_{\text {width }}^{B H}=2.1$

Figure 14 shows the standard deviation of the estimated delay error at the output of the DLL, with the different windowing techniques. It considers tracking conditions in a single-tap channel, where the only 
impairment is thermal noise. A second order loop is used, with the noise equivalent loop bandwidth set at $10 \mathrm{~Hz}$.

The DLL theoretical tracking performances are very good. For the DVB-SH signal parameters, $N_{F F T}=$ $2048, C P=1 / 4$ and $B=5 \mathrm{MHz}$, the sample duration is $175 \mathrm{~ns}$, corresponding to $52.5 \mathrm{~m}$. Therefore, a sub-meter standard deviation is obtained for SNR above $-10 \mathrm{~dB}$. In a typical DVB-SH deployment, the expected minimum SNR is likely to be around $0 \mathrm{~dB}$, and often well above.

Also, as expected, the use of strong sidelobe-attenuating window slightly degrades the accuracy, but the degradation should be negligible compared to other sources of errors such as NLOS tracking or close multipath.

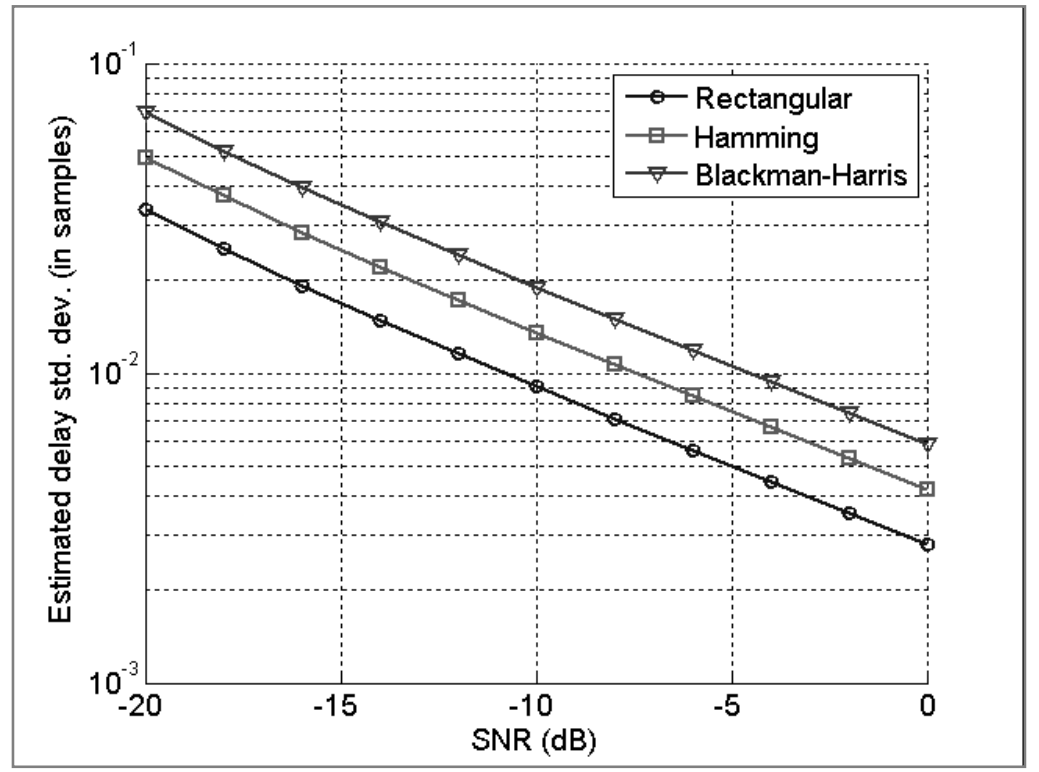

Figure 14 - Tracking loop error standard deviation vs SNR

\section{Multipath error enveloppe}

Finally, the multipath error envelope for windowed DLL is shown in Figure 15 for the 3 considered windows. The multipath error envelope is often used in order to assess the DLL sensitivity to multipath. This plot consists of the collection of the discriminator's outputs for a single multipath channel that has an amplitude half of the direct signal and a delay that takes different values. To have the extreme values of the error, the multipath is considered in phase and in phase opposition with the direct signal. This analysis is done without noise.

As expected, the windowed DLLs are more affected by close multipath, due to the larger width of the correlation main lobe.

Also, windowed DLLs are no longer affected by far multipath, due to the removal of sidelobes.

With a sample length of $52.5 \mathrm{~m}$, the error due to close multipath may reach up to $25 \mathrm{~m}$ in the conditions of this figure (2 multipath with relative amplitude equal to 2 ). 


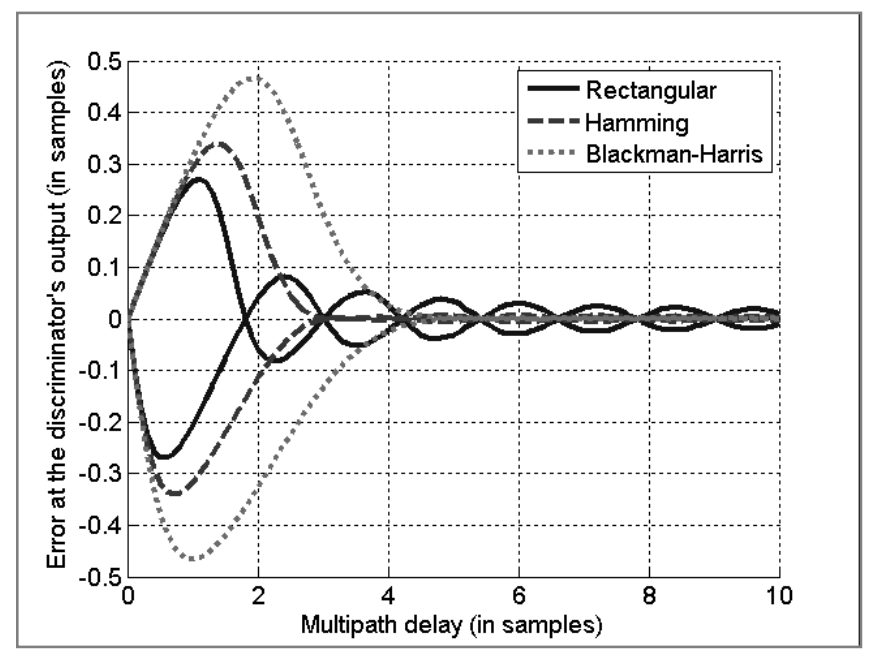

Figure 15 - Multipath error envelope for windowed DLL

\section{Multipath delay clustering for emitter discrimination}

All multipaths associated to one emitter should be in the same delay range. For example, most of the significant multipaths measured in the presented SFN CIR time series arrive within $2 \mu \mathrm{s}$. There may be very particular cases creating larger multipath delays, such as far high-rise obstacles (skyscrapers, surrounding mountains) but they will not be considered in this study.

It is therefore possible to cluster the different estimated delays in order to decide if they are associated to the same emitter. To do this, classic clustering algorithms have been used. They have been implemented from conventional techniques found in [27].

At the input of the emitter discrimination process, $N_{\text {delay }}$ estimated delay are available, noted

$$
\left\{\tau_{1}, \tau_{2}, \ldots, \tau_{N_{\text {delay }}}\right\}
$$

A hierarchical cluster tree is created from these values, following these steps:

1. Pairwise euclidian distances are calculated between each mean delay value:

$$
d\left(\tau_{n}, \tau_{m}\right)=\left|\tau_{n}-\tau_{m}\right|
$$

2. A cluster is formed by the two elements having the minimum distance between them. The 2 grouped elements (say elements $p$ and $q$ ) are replaced by a new one associated to the mean delay of the 2 grouped elements. $\tau_{\text {new element }}=\frac{1}{2}\left(\tau_{p}+\tau_{q}\right)$

3. Steps 1 and 2 are repeated until all elements are clustered.

The final clusters are then formed by choosing a threshold on the distance separating 2 branches. Obviously, this threshold should be above the maximum delay of signal replicas coming from a single emitter, ie delays below this value will be part of the same cluster. A second constraint on the choice of the clustering threshold is that its value should be below the minimum delay observed between signal replicas from 2 different emitters. This second constraint will therefore depend on the introduced artificial delay between emitters.

Once this threshold is decided, the clusters are determined when 2 branches are separated by a distance above this threshold. 


\section{Simulation results using real SFN CIR measurements}

\section{Simulation of the pseudo-range estimation technique}

The pseudo-range estimation strategy consists in acquiring and tracking in parallel the delay of all the significant received signal replicas. The shortest delay of a specific cluster is then selected to compute the pseudorange associated with the corresponding emitter. This is done in order to increase the chances of tracking the LOS replica, which may be received with lower power than a later replica.

In order to improve the chance of acquiring (or re-acquiring) the desired LOS replica, delay acquisition by Matching Pursuit is launched periodically. The time between 2 acquisitions has been empirically set to $14.3 \mathrm{~s}$, as it has been observed as a good compromise between computational load and LOS successful acquisition.

Finally, in order to keep the number of running DLLs low, some DLL exclusion schemes have been set up. Without going into further implementation details, the DLL exclusion processes are set up in the following conditions:

- if 2 DLL are tracking the same delay;

- if 1 DLL is diverging (due to the disappearing of a signal replica for example);

- if the signal replica power is too low.

The proposed delay estimation and delay clustering techniques have been applied to a DVB-SH signal filtered by the presented SFN CIR measurement. 3 simulations were done, using the different windowing techniques. The common parameters are collected in Table 1.

\begin{tabular}{|c|l|}
\hline$N_{F F T}$ & 2048 \\
\hline$C P$ & $1 / 4$ \\
\hline$B$ & $5 \mathrm{MHz}$ \\
\hline Emitter EIRP & $53.2 \mathrm{dBm}[12]$ \\
\hline Noise floor level & $-102.6 \mathrm{dBm}[10]$ \\
\hline SNR & Between 9.2 and $49.2 \mathrm{~dB}$ \\
\hline$B_{l}$ & $10 \mathrm{~Hz}$ \\
\hline$T_{i}$ & $T_{\text {symb }}=448 \mu \mathrm{s}$ \\
\hline Time between 2 acquisition phases & $T_{A C Q}=14.336 \mathrm{~s}$ \\
\hline Acquisition threshold & $-120 \mathrm{dBm}$ \\
\hline Tracking threshold & $-140 \mathrm{dBm}$ \\
\hline Clustering threshold & $2.5 \mu \mathrm{s}$ \\
\hline Maximum number of DLL launched after each acquisition & 15 \\
\hline
\end{tabular}

Table 1 - Simulation parameters

For each simulation, the plot of the error between the estimated delay of each cluster and the delay of the true first path of each emitter is shown (Figure 17, Figure 18, Figure 19). Additionally, the plot of the estimated delays provided by each DLLs (after clustering) is shown only for the case without windowing (Figure 16).

Besides, statistics on the availability of the pseudo-range and on the mean error and the standard deviation of the error for each emitter will be collected in tables. Two values of the mean and the standard deviation of the error are calculated: the first one is computed over the whole time series. The second one is the median of the local mean and standard deviation of the error, computed over slices of the time series lasting 14,3 s. This second value permits to have an idea of the performance reached for $50 \%$ of the time. 


\section{Rectangular window}

\begin{tabular}{|l|c|c|c|c|c|}
\hline & $\begin{array}{c}\text { Availability } \\
\text { (\%) }\end{array}$ & \multicolumn{2}{|c|}{ Mean error (m) } & \multicolumn{2}{c|}{ Error standard deviation (m) } \\
\hline Emitter \#1 & 100 & -185.2 & 0.04 & 288.9 & median \\
\hline Emitter \#2 & 100 & -9.7 & 0.98 & 144.3 & 11.5 \\
\hline Emitter \#3 (sat) & 67.1 & 11.2 & 0.02 & 62.8 & 1.98 \\
\hline
\end{tabular}

Table 2 - Simulation results for the rectangular window

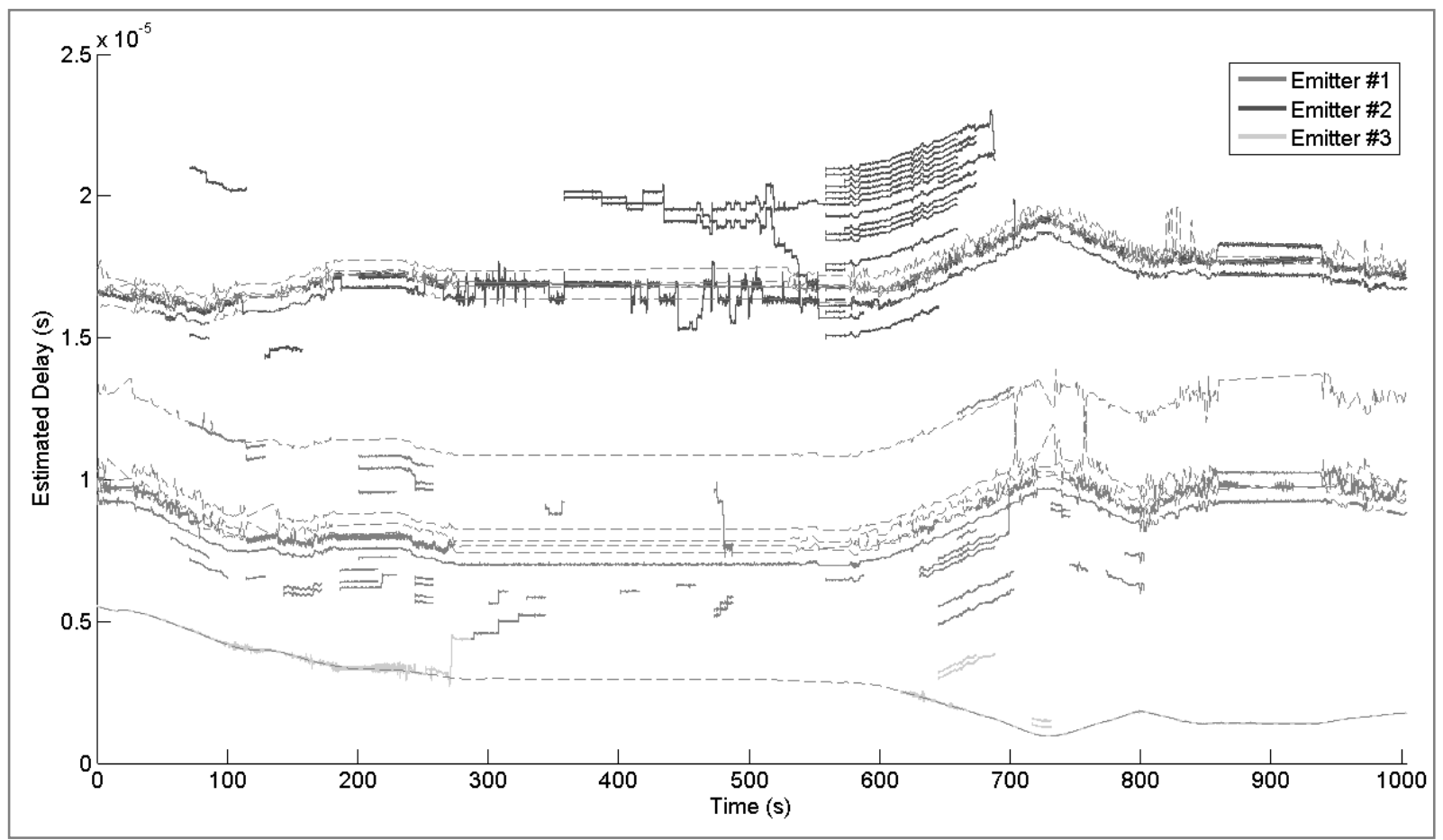

Figure 16 - Estimated multipath delay coming from all the operating DLLs after clustering (colored) for the rectangular window simulation. The gray lines represent the true delays.

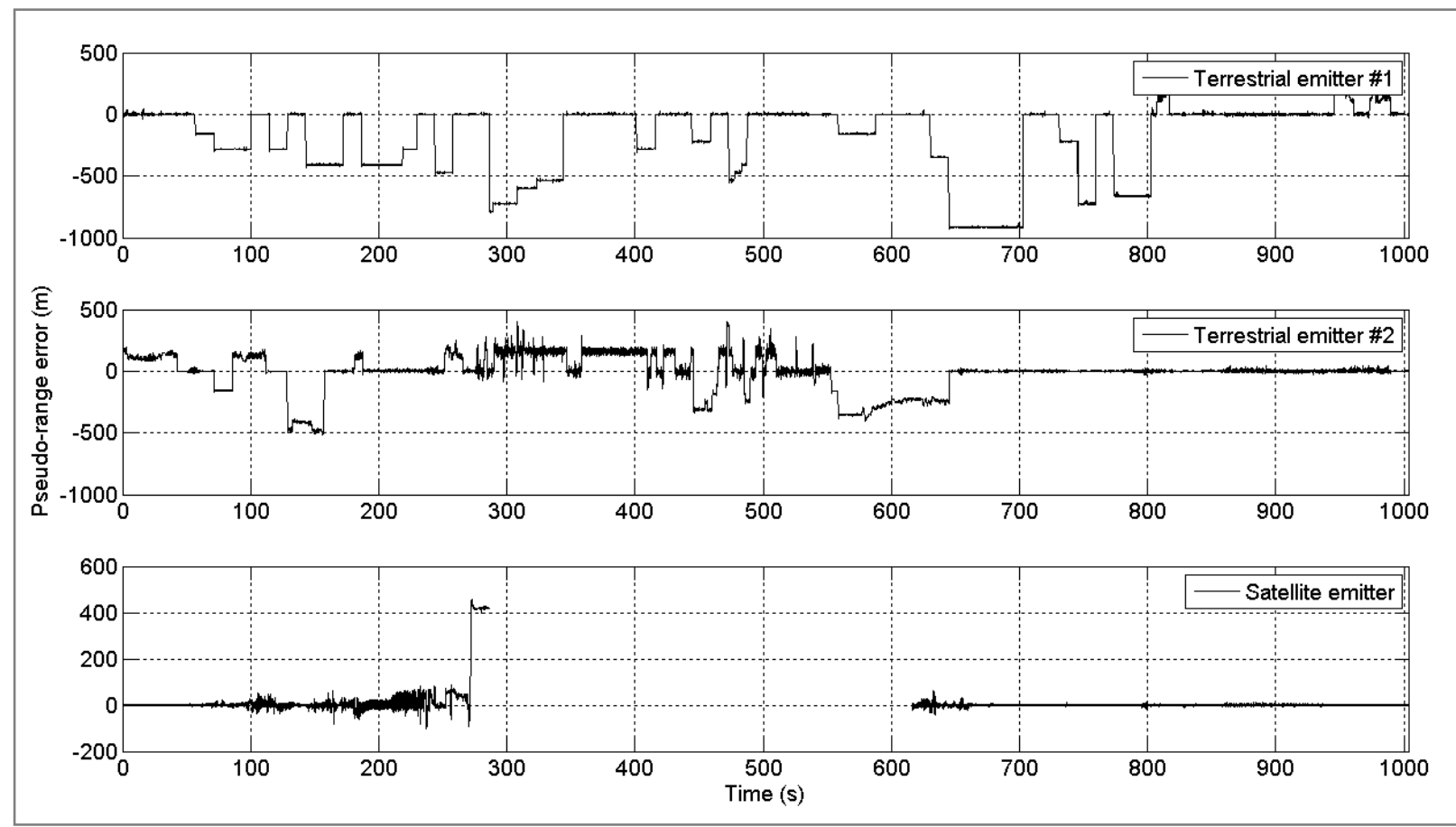

Figure 17 - Pseudo-range error time series for each emitter for the rectangular window simulation 


\section{Hamming window}

\begin{tabular}{|l|l|l|l|l|l|}
\hline & \multicolumn{1}{|c|}{$\begin{array}{c}\text { Availability } \\
(\mathbf{\%})\end{array}$} & \multicolumn{2}{c|}{ Mean error $(\mathbf{m})$} & \multicolumn{2}{c|}{ Error standard deviation (m) } \\
\hline & 100 & -150.2 & -0.06 & 219.1 & median \\
\hline Emitter \#1 & 100 & 26.7 & 0.41 & 61.9 & 2.5 \\
\hline Emitter \#2 & 88.6 & 29.7 & 0.02 & 169.0 & 1.9 \\
\hline Emitter \#3 (sat) & 8.6 &
\end{tabular}

Table 3 - Simulation results for the Hamming window

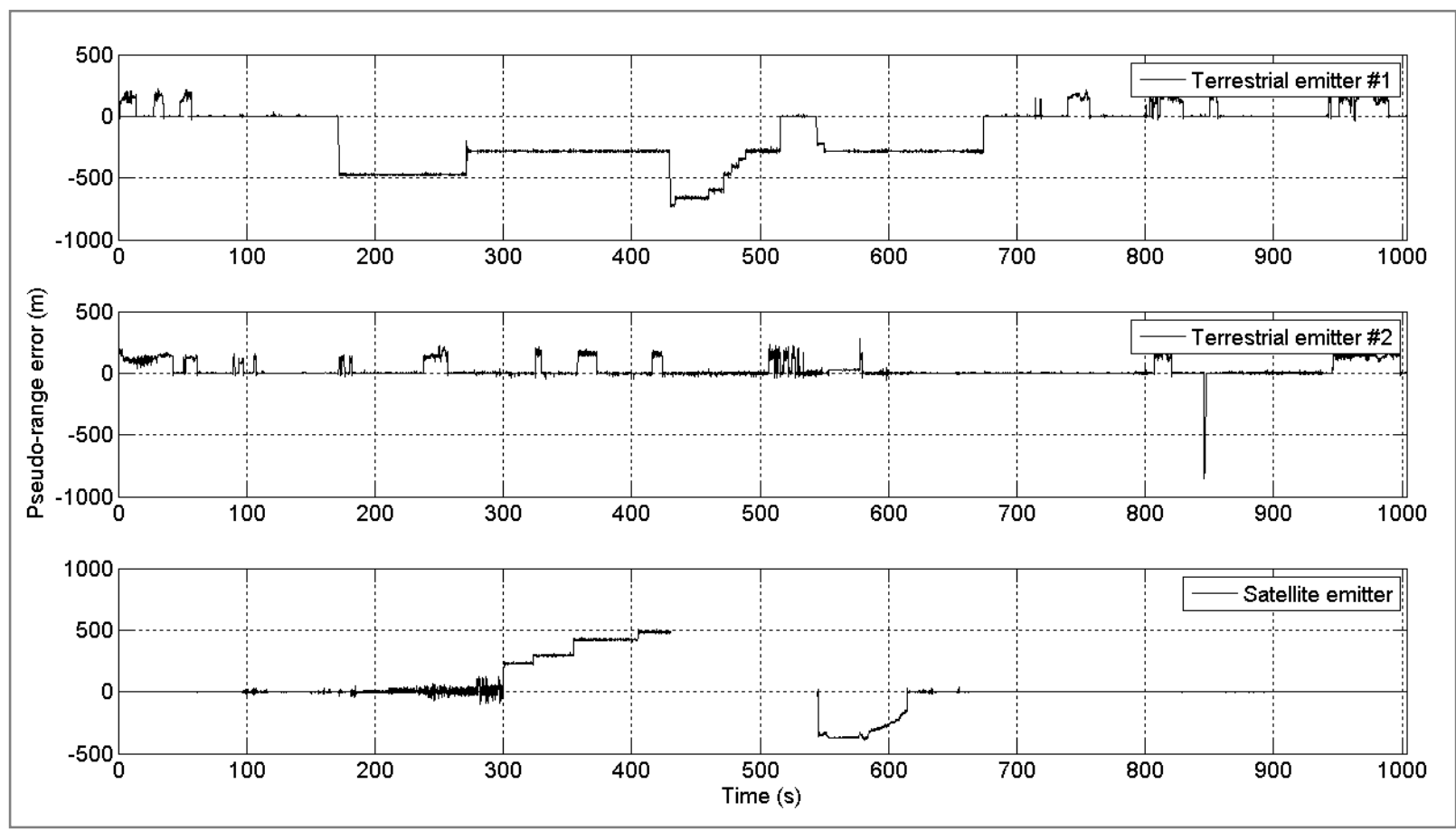

Figure 18 - Pseudo-range error time series for each emitter for the Hamming window simulation

\section{Blackman-Harris window}

\begin{tabular}{|l|l|l|l|l|l|}
\hline & \multirow{2}{*}{$\begin{array}{c}\text { Availability } \\
(\mathbf{\%})\end{array}$} & \multicolumn{2}{c|}{ Mean error (m) } & \multicolumn{2}{c|}{ Error standard deviation (m) } \\
\cline { 3 - 6 } & \multicolumn{2}{|c|}{ overall } & \multicolumn{1}{c|}{ median } & \multicolumn{1}{c|}{ overall } & \multicolumn{1}{c|}{ median } \\
\hline Emitter \#1 & 98.6 & 35.0 & 8.3 & 80.5 & 32.4 \\
\hline Emitter \#2 & 92.9 & 62.0 & 70.4 & 79.7 & 51.1 \\
\hline Emitter \#3 (sat) & 100 & 0.003 & 0.000 & 0.9 & 0.7 \\
\hline
\end{tabular}

Table 4 - Simulation results for the Blackman-Harris window 


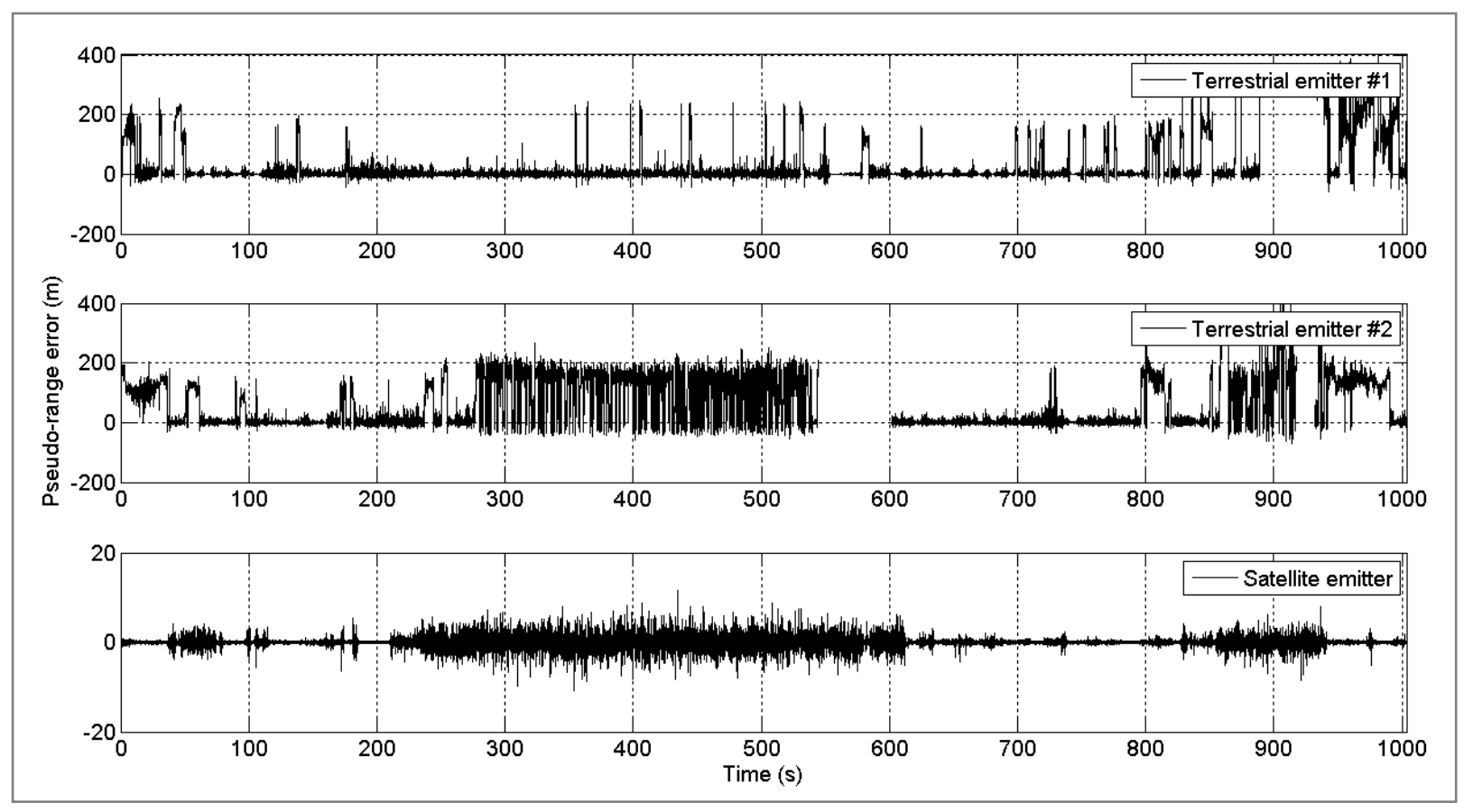

Figure 19 - Pseudo-range error time series for each emitter for the Blackman-Harris window simulation

\section{Conclusion on the pseudo-range estimation performances}

The errors on the pseudo-range estimation of each emitter are mainly affected by 3 phenomena:

- An unavailability of the pseudo-range due to the Near-Far effect, which greatly affects the pseudo-range estimation to the satellite emitter for the rectangular window. This unavailability period is reduced by using a Hamming window and completely solved with the Blackman-Harris window, thanks to the reduction of the sidelobes created by emitter \#1 (the closest and thus strongest emitter) in the CIR estimate.

- A large negative error for the terrestrial emitters due to the tracking of early strong sidelobes present in the CIR estimate. This affects both terrestrial emitters in the rectangular window simulation, while only the strongest emitter is affected in the Hamming window simulation. The Blackman-Harris window simulation is not affected by this phenomenon, again thanks to the strong attenuation of the sidelobe permitted by this window.

- Numerous leaps of the pseudo-range errors for the Blackman-Harris window simulation, due to the presence of significant close multipath coming from the same emitter. The delay estimation process goes from one multipath to the other depending on the fading condition of each multipath. This phenomenon is also favored because of the main lobe widening induced by the use of the Blackman-Harris window.

The best pseudo-range estimation performance is obtained when using a windowing technique. Good results are obtained for median values, which corresponds to the performances reached for $50 \%$ of the time. For example, the Hamming window simulation shows that all pseudo-ranges are estimated with a near $0 \mathrm{~m}$ bias and a standard deviation below $4 \mathrm{~m}$ for at least $50 \%$ of the time. However, early sidelobe tracking or close multipath tracking are creating local pseudo-range errors of several hundred meters.

The use of strong sidelobe-attenuating window is beneficial in order to make the delay estimation from low-power emitter possible. However, the main lobe widening induced by such window makes it quite sensitive to close significant multipath from the same emitter, which is translated in numerous error leaps of several hundred meters. One way to improve this situation would be to switch between 2 windowing techniques, depending on the tracking conditions. 
Also, the impact of the DLL noise equivalent loop bandwidth was not studied. In these simulations, a bandwidth of $10 \mathrm{~Hz}$ was chosen in order to let the DLL follow the delay variation. By reducing this bandwidth, the receiver may be able to stay on the same multipath delay affected by fast fading long enough to avoid being attracted by another signal replica.

\section{Results in the position domain}

In the previous simulation, the pseudo-range error was computed as the difference between the selected delay estimate and the first multipath present in the measurement. Therefore, the non-line-of-sight (NLOS) conditions have not been taken into account: ie if a pseudo-range error is $0 \mathrm{~m}$, it means that the first multipath delay is perfectly estimated, but not that this first multipath delay corresponds to the lineof-sight (LOS) signal.

The impact of the NLOS conditions can be indirectly observed by computing the resulting position from the estimated pseudo-ranges and comparing it to a GPS position recorded during the measurement campaign.

Also, the user position may be affected by the geometric configuration of the receiver and emitters location, through the well-known Dilution of Precision (DoP) factors [28]. In the simulation, only 3 emitters are present, and they are also aligned, which will imply a high DoP value, and therefore a degraded user position.

The position calculation was done using a conventional non-linear least square algorithm [28]. The estimated pseudo-ranges were associated to each emitter manually, but the introduction of artificial delay should provide a means to automate this. Only the $2 \mathrm{D}$ position was computed, the clock offset between the receiver and the emitters being simulated and assumed constantly null-valued. The position is estimated for every transmitted OFDM symbol (with a rate of $2250 \mathrm{~Hz}$ ), but the shown position is smoothed using a running average over $1 \mathrm{~s}$.

Figure 20 shows the position computed from a perfect estimation of the delay of the first multipath from each emitter. The norm of the position error is shown in Figure 21. While the trace obtained with the DVB-SH measurements are approximately following the GPS trace, there is a position error which can reach up to $120 \mathrm{~m}$, with a mean absolute error of $24.3 \mathrm{~m}$. The error is induced by potential measurement errors at the channel sounding device level and also due to NLOS conditions. However, this DVB-SH trace should be considered as the best reachable performances with this SFN CIR measurement set.

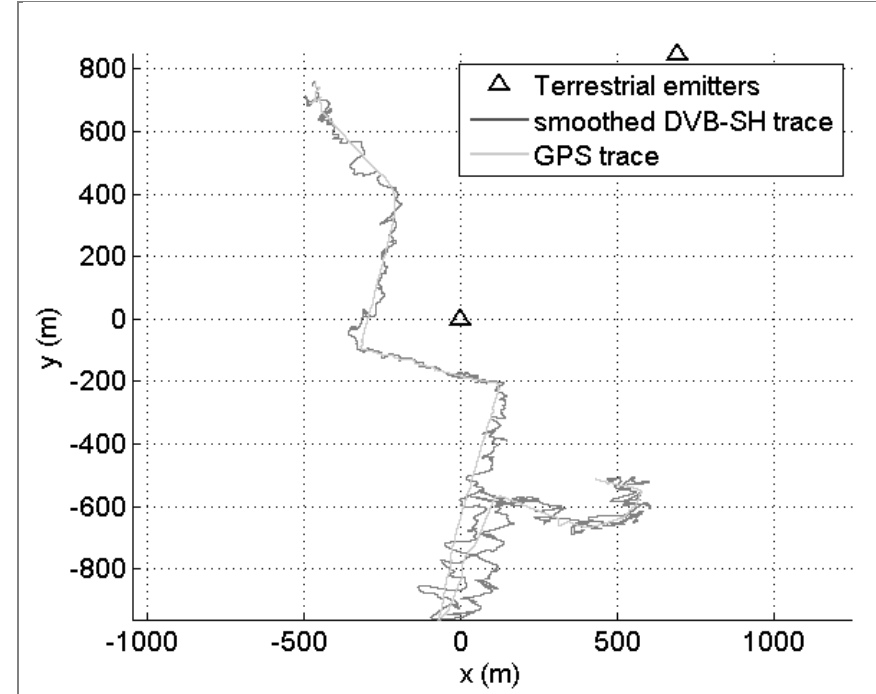

Figure 20 - Estimated and reference trace using perfectly estimated pseudo-range measurements

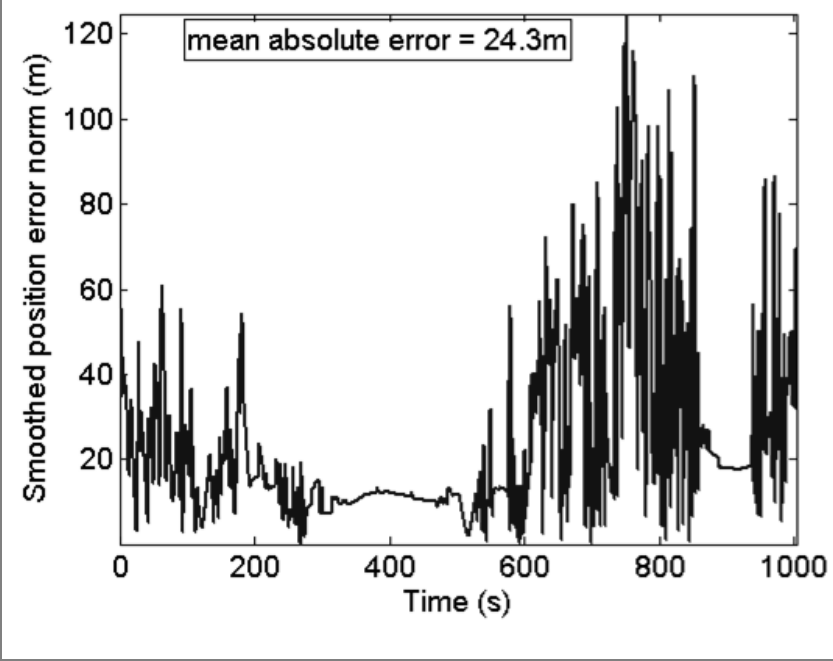

Figure 21 - Position error norm using perfectly estimated pseudo-ranges measurements 
Figure 22 shows the same results with the pseudo-range estimated with the presented method and using the Blackman-Harris window. Compared to the reference DVB-SH trace, some significant additional errors can be observed notably toward the end of the trace. They correspond to instants where later multipath delays are tracked instead of the first present multipath. The maximum error reaches $700 \mathrm{~m}$ and the mean absolute error is $93.8 \mathrm{~m}$ on the overall time series. By calculating the mean on the first $800 \mathrm{~s}$ of the error time series, we would obtain a mean absolute error of $63.7 \mathrm{~m}$.

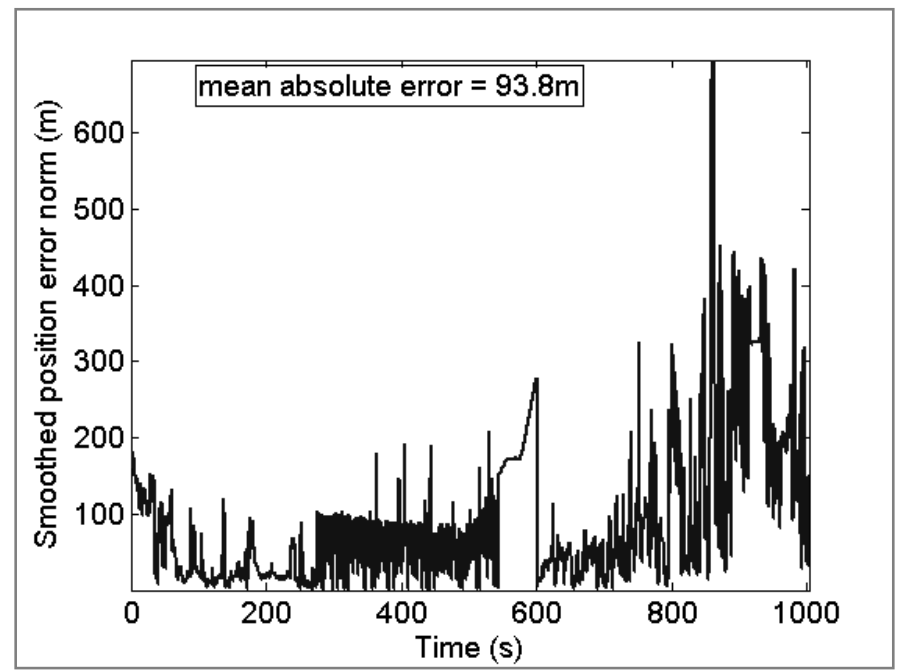

Figure 22 - Position error norm using estimated pseudo-ranges measurements using a Blackman-Harris window

Figure 23 shows the same results with the best estimated pseudo-ranges among all presented simulations. Pseudo-range estimates from emitter \#1 and \#3 are taken from the Blackman-Harris window simulation, while pseudo-range estimates from emitter \#2 are taken from the Hamming widow simulation, which is much less affected by later multipath tracking. The maximum error reaches $700 \mathrm{~m}$ and the mean absolute error is $76.8 \mathrm{~m}$. By calculating the mean on the first $800 \mathrm{~s}$ of the error time series, we would obtain a mean absolute error of $41.9 \mathrm{~m}$, which is quite interesting when looking at the low number of emitters considered and the very adverse location of these emitters.

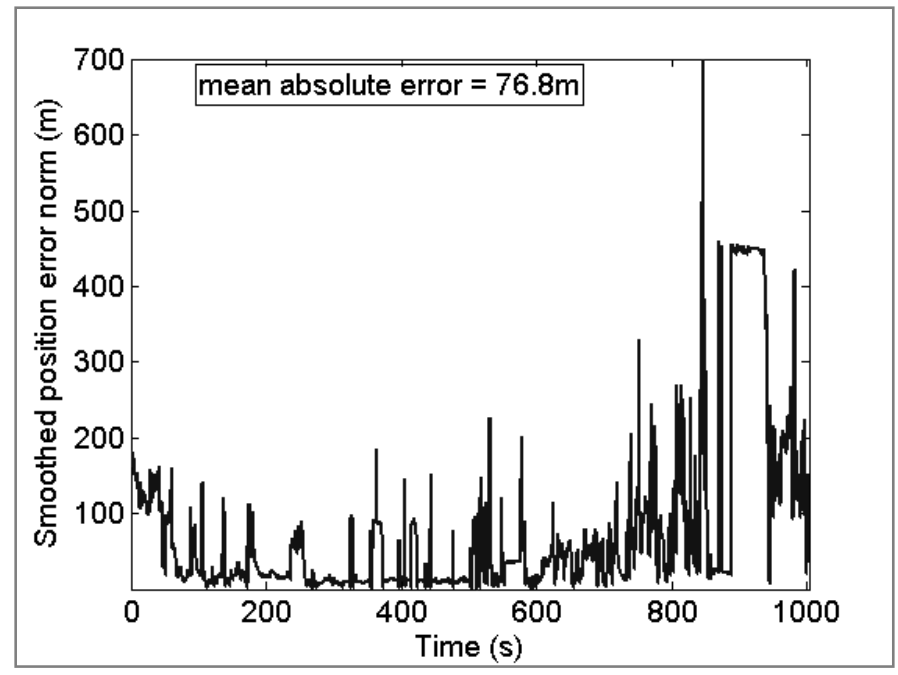

Figure 23 - Position error norm using best combined estimated pseudo-ranges measurements

\begin{tabular}{|c|c|c|}
\hline Simulation & $\begin{array}{c}\text { Mean position error for } \\
\text { the overall time series }\end{array}$ & $\begin{array}{c}\text { Mean position error } \\
\text { for the first 800s }\end{array}$ \\
\hline Ideal pseudo-range estimates & $24.3 \mathrm{~m}$ & $22.5 \mathrm{~m}$ \\
\hline
\end{tabular}




\begin{tabular}{|l|c|c|}
\hline Blackman-Harris pseudo-ranges & $93.8 \mathrm{~m}$ & $63.7 \mathrm{~m}$ \\
\hline $\begin{array}{l}\text { Combined Blackman-Harris / } \\
\text { Hamming pseudo-ranges }\end{array}$ & $76.8 \mathrm{~m}$ & $41.9 \mathrm{~m}$ \\
\hline
\end{tabular}

Table 5 - Positioning performances obtained by the proposed method

\section{Conclusion and perspectives}

Positioning using a DVB-SH systems presents some advantages: the high expected received signal power should provide a good positioning service coverage in metropolitan centers or even indoor, and the emitter network is synchronized thus permitting to use time of arrival timing measurements for pseudo-range measurements. Some challenges associated with the choice of DVB-SH is the configuration of the emitter network in SFN, making emitter discrimination and identification challenging. Also, the urban propagation environment may greatly degrade the pseudo-range estimation, by masking the LOS signal or due to the huge signal power variations. Also, Near-Far effect, ie the jamming of remote emitters by one closer emitter, appears due to the use of a terrestrial network of emitter.

In this paper, we briefly recalled a system-level modification to permit emitter discrimination and identification. This modification consists in introducing different artificial delay at the emission of the signal by the emitters, so no signal replicas from different emitters arrive at the same delay all over the service coverage. This modification will not impact the telecommunication service as long as the correct signal parameters are chosen.

Then, signal processing techniques are presented to extract pseudo-ranges from the received DVB-SH signal. DVB-SH uses the particular OFDM modulation to fight against multipath. The proposed technique uses the pilot symbols already present in the DVB-SH signal to obtain a CIR estimate. This CIR estimate may benefit from windowing techniques in order to attenuate the sidelobes present in the correlation peak shape, and also to mitigate the Near-Far effect. Then, Matching Pursuit algorithm is used to initialize several DLLs, which are launched in parallel. After clustering the delay estimate to group them by emitter of origin, the earliest delay is used to compute a pseudo-range. In order to increase the chance of tracking the earliest multipath coming from each emitter, the Matching Pursuit algorithm is re-started periodically.

This method has been tested using SFN CIR measurement taken from a real channel sounding campaign done in a urban environment. The use of windowed correlation greatly improves the availability and the accuracy of the pseudo-ranges estimates. However, the pseudo-range estimates are affected by important leaps due to the tracking of later multipaths. The error in the position domain was obtained thanks to the use of a conventional non-linear least square algorithm, which showed a mean positioning error around $40 \mathrm{~m}$ using the best pseudo-range estimation methods. Doing the same simulations with a larger signal bandwidth may bring increased performances, both in signal tracking accuracy and in robustness against multipaths. Indeed, the simulations presented here uses a signal bandwidth of $5 \mathrm{MHz}$, while the DVB-SH standards provisions bandwidth up to $8 \mathrm{MHz}$. Also, the test configuration was far from optimal since a realistic deployment of DVB-SH emitters should allow to view a significantly larger number of emitter [18].

It is important to realize that this work showed the feasibility of positioning using DVB-SH signals only. Obviously, this work would clearly benefit from additional measurement campaigns, so that the parameters used in the proposed methods can be tuned to different types of urban environment, and to assess the positioning performances in presence of more emitters. On the signal processing level, techniques for choosing the right window to be applied depending on the multipath configuration would greatly improve the pseudo-range estimation. Looking at the pseudorange error plots, it seems feasible to detect the large leaps of the estimated delay during the tracking phase, which could be the basis for mitigating this most important source of positioning error. Also, another solution to be more resistant to these pseudorange jumps would be to use a DLL noise bandwidth significantly smaller (around $1 \mathrm{~Hz}$ ). Finally, this method would greatly benefit from more complex position solving techniques, which could implement multipath rejection/mitigation techniques, reliability tests, and coupling with GPS or inertial measurements. 
As a conclusion, these first results, combined with the ideas for further enhancement of DVB-SH positioning, provide a positive view on the opportunity to use DVB-SH as a source for indoor and deep urban positioning.

\section{References}

[1] FCC. 2000. OET Bulletin No. 71 - Guidelines for Testing and Verifying the Accuracy of Wireless E911 Location Systems.

[2] Raquet JF, Miller MM, Nguyen TQ. Issues and Approaches for Navigation Using Signals of Opportunity. In: ION NTM 2007; 2007. p. 1073-1080.

[3] Zhao Y. Standardization of mobile phone positioning for 3G systems. Communications Magazine, IEEE. 2002;40(7):108-116.

[4] Ludden B, Lopes L. Cellular based location technologies for UMTS: a comparison between IPDL and TAIPDL. IEEE VTC 2000.

[5] Porcino D. Performance of a OTDOA-IPDL positioning receiver for 3GPP-FDD mode. In: 3G Mobile Communication Technologies, 2001. 2001. p. 221-225.

[6] Rabinowitz M, Spilker JJ. A new positioning system using television synchronization signals. Broadcasting, IEEE Transactions on. 2005;51(1):51-61.

[7] Hein G, Eissfeller B, Oehler V, Winkel JO. Synergies between Satellite Navigation and Location Services of Terrestrial Mobile Communication. In: ION GPS 2000; 2000. p. 535-544.

[8] Palmer D, Moore T, Hill C, Andreotti M, Park D. Non-GNSS Radio Positioning using the Digital Audio Broadcasting (DAB) Signal. In: ION GNSS 2009; 2009. p. 864-872.

[9] Final draft ETSI EN 302583 v1.1.0, Digital Video Broadcasting (DVB); Framing Structure, channel coding and modulation for Satellite Services to Handheld devices (SH) below $3 \mathrm{GHz}$. January 2008

[10] ETSI TS 102584 v1.1.1. Digital Video Broadcasting (DVB); DVB-SH Implementation Guidelines. December 2008.

[11] Laine P. Alcatel-Lucent's proposal for unlimited mobile TV: a hybrid satellite/terrestrial broadcast network based on DVB-SH; 2007.

[12] Chuberre N, Bodevin F, Courseille O, Duval R, Dussauby E, Selier C. Unlimited Mobile TV. In: ASMS 2006

[13] Bingham JAC. Multicarrier modulation for data transmission: an idea whose time has come. Communications Magazine, IEEE. 1990; 28(5):5-14.

[14] van de Beek JJ, Sandell M, Borjesson PO. ML estimation of time and frequency offset in OFDM systems. Signal Processing, IEEE Transactions on. 1997;45(7):1800-1805.

[15] Mattsson A. Single frequency networks in DTV. Broadcasting, IEEE Transactions on. 2005 November;51(4):413-422.

[16] Pérez Fontán F, Mariño Espiñeira P. Modeling the Wireless Propagation Channel - A Simulation Approach with Matlab. 1st ed. Wiley; 2008.

[17] Thevenon P, Julien O, Macabiau C, Serant D, Ries L, Corazza S, et al. Positioning principles with a mobile TV system using DVB-SH signals and a Single Frequency Network. In: 2009 16th International 
Conference on Digital Signal Processing. IEEE; 2009. p. 1-8.

[18] PhD Manuscript. "S-band air interfaces for navigation systems: a focus on OFDM signals". 2010.

[19] Lacoste F, Carvalho F, Scot G, Ros B. Measurements of the macrocell propagation channel at 2.2 and 3.8 $\mathrm{GHz}$ and comparison with some analytical models. In: COST Action IC0802; 2009.

[20] Serant D, Julien O, Macabiau C, Thevenon P, Dervin M, Corazza S, et al. Development and Validation of an OFDM/DVB-T Sensor for Positioning. In: IEEE/ION PLANS 2010; 2010. p. 988-1001.

[21] Cotter SF, Rao BD. Sparse channel estimation via matching pursuit with applicaion to equalization. Communications, IEEE Transactions on. 2002;50(3):374-377.

[22] Thevenon P, Julien O, Macabiau C, Serant D, Corazza S, Bousquet M, et al. Pseudo-Range Measurements Using OFDM Channel Estimation. In: ION GNSS 2009; 2009. p. 481-493.

[23] Fleury BH, Tschudin M, Heddergott R, Dahlhaus D, Ingeman Pedersen K. Channel parameter estimation in mobile radio environments using the SAGE algorithm. IEEE Journal on Selected Areas in Communications. 1999 March;17(3):434-450.

[24] Paulraj A, Roy R, Kailath T. Estimation Of Signal Parameters Via Rotational Invariance Techniques Esprit. In: Circuits, Systems and Computers, 1985. Nineteeth Asilomar Conference on; 1985. p. 83-89.

[25] Yang B, Letaief KB, Cheng RS, Cao Z. An improved combined symbol and sampling clock synchronization method for OFDM systems. In: Wireless Communications and Networking Conference; 1999. p. 1153-1157.

[26] Stephens SA, Thomas JB. Controlled-root formulation for digital phase-locked loops. Aerospace and Electronic Systems, IEEE Transactions on. 1995;31(1):78-95.

[27] Jain A, Dubes R. Algorithms for Clustering Data. Upper Saddle River, NJ: Prentice-Hall. 1988.

[28] Kaplan ED, Hegarty C. Understanding GPS: Principles and Applications. 2nd ed. Artech House Publishers; 2005. 